\title{
Relativistic theory of magnetic scattering of $x$ rays: Application to ferromagnetic iron
}

\author{
E. Arola and P. Strange \\ Physics Department, Keele University, Keele, Staffordshire ST5 5BG, United Kingdom \\ B. L. Gyorffy \\ H.H. Wills Physics Laboratory, Bristol University, Tyndall Avenue, Bristol BS8 1TL, United Kingdom
}

(Received 29 August 1996)

\begin{abstract}
We present a detailed description of a first-principles formalism for magnetic scattering of circularly polarized $x$ rays from solids in the framework of the fully relativistic spin-polarized multiple-scattering theory. The scattering amplitudes are calculated using a standard time-dependent perturbation theory to second order in the electron-photon interaction vertex. Particular attention is paid to understanding the relative importance of the positive- and negative-energy solutions of the Dirac equation to the scattering amplitude. The advantage of the present theory as compared with other recent works on magnetic x-ray scattering is that, being fully relativistic, spin-orbit coupling and spin-polarization effects are treated on an equal footing. Second, the electron Green's function expressed in terms of the path operators in the multiple-scattering theory allows us to include the contribution of the crystalline environment to the scattering amplitude. To illustrate the use of the method we have done calculations on the anomalous magnetic scattering at the $K, L_{\mathrm{II}}$, and $L_{\mathrm{III}}$ absorption edges of ferromagnetic iron. [S0163-1829(97)00501-8]
\end{abstract}

\section{INTRODUCTION}

In spite of the early predictions of light scattering from magnetic moments of spin-1/2 particles by Low, ${ }^{1}$ and GellMann and Goldberger, ${ }^{1}$ and, later on, Platzman and Tzoar $^{2}$ who first proposed the use of $x$-ray-scattering techniques to study magnetization densities in solids, progress in magnetic structure studies using $\mathrm{x}$ rays has been limited by the fact that the magnetic $\mathrm{x}$-ray-scattering cross section is smaller than that for charge scattering by the order of $\left(\hbar \omega / m c^{2}\right)^{2} \cdot{ }^{3,4}$ It was not until recently that de Bergevin and Brunel $^{5}$ demonstrated experimentally the effect of magnetism on x-ray scattering in solids. And it was only after the observation of a huge resonant enhancement in the x-rayscattering cross section of rare-earth metal holmium by Gibbs et al. ${ }^{6}$ that has launched a great interest in using x-ray magnetic scattering as a new experimental tool to investigate magnetic properties of solids. Kao et al. have observed a similar effect also at the transition metal $\operatorname{iron}^{7}$ and cobalt ${ }^{8}$ $L_{\text {II,III }}$ edges for a thin film structure.

Furthermore, with the presently available third-generation high-intensity, high-resolution synchrotron radiation sources magnetic x-ray-scattering studies have become feasible. Especially, when the photon energy is tuned through an absorption edge of a constituent, a large and species selective enhancement of the scattering cross section occurs (resonant, anomalous scattering). Remarkably, using polarization properties of $\mathrm{x}$ rays it is possible to distinguish between orbital and spin scattering, unlike in the neutron case.

Some of the recent theoretical and experimental work using linearly or circularly polarized $\mathrm{x}$ rays for studying magnetic phenomena in condensed matter will be briefly reviewed in the following. For a nonrelativistic many-electron system interacting with a quantized electromagnetic field, using perturbation theory, Blume $^{3}$ has derived a general expression for the $\mathrm{x}$-ray magnetic-scattering cross section, which allows the electron binding effects to be accounted for. Later on Blume and Gibbs ${ }^{9}$ have further developed the formula such that, for example, the orbital and spin angular momentum contributions of both ferromagnets and antiferromagnets can be separately measured in a variety of geometries. Hannon et al. ${ }^{10}$ have presented a nonrelativistic theory of $\mathrm{x}$-ray resonance exchange scattering with explicit formulas for the electric dipole $(E 1)$ and quadrupole $(E 2)$ contributions. Using this theory, Fasolino et al. ${ }^{11}$ have investigated magnetism in antiferromagnetic UAs(001) and ferromagnetic $\mathrm{Gd}(0001)$ surfaces. Combining the $\mathrm{x}$-ray resonant magnetic scattering with $\mathrm{x}$-ray surface diffraction, they have found that the diffraction spectra for linearly polarized light are sensitive to orientation and magnitude of the surface magnetic moments. Although Rennert ${ }^{12}$ has presented a semirelativistic magnetic x-ray-scattering theory in terms of the Green's functions, which could be calculated from first principles, to date no such calculations have been performed.

Current experimental interest in using magnetic effects in x-ray scattering is highlighted by the work of Hannon et al. ${ }^{10}$ Gibbs et al. ${ }^{6}$ Lang et al.,${ }^{13}$ Giorgetti et al.,${ }^{14}$ and Hill et al..$^{15}$ to mention but a few. The theory we shall present aims to aid the interpretation of these experiments in addition to suggesting new useful measurements.

In Sec. II we present a detailed description of our formal first-principles theory of magnetic scattering of $\mathrm{x}$ rays based on a fully relativistic spin-polarized multiple-scattering theory using the time-dependent perturbation theory. We illustrate its use with a calculation of resonant scattering at the $L$ and $K$ absorption edges of ferromagnetic iron in Sec. III. The advantages of the present theory compared to earlier ones $^{3,9,16,12}$ are that being fully relativistic it treats spin-orbit coupling and spin-polarization effects on an equal footing. Notably, our theory includes the contribution of the crystalline environment to the scattering amplitude in a manner which allows an accurate implementation of the theory. 


\section{OUTLINE OF THE RELATIVISTIC MAGNETIC X-RAY-SCATTERING THEORY}

Here we describe the details of our formal first-principles theory on the magnetic scattering of $x$ rays from solids. It is based on the fully relativistic spin-polarized multiplescattering theory ${ }^{17-19}$ in conjunction with the time-dependent perturbation theory. ${ }^{20}$ To simplify the presentation a straightforward canonical perturbation theory will be used instead of the more sophisticated Keldysh diagram method. ${ }^{21,22}$ In Secs. II $\mathrm{B}$ and II C we derive the general expressions for the scattering amplitudes involving positive- and negative-energy states of the Dirac Hamiltonian, respectively. In Sec. II D we present the relevant equations for a special case of magnetic scattering from perfect crystals, i.e., the magnetic Bragg scattering, and in Sec. II E we define dichroism from the viewpoint of magnetic-scattering experiments.

\section{A. Basic background}

In what follows we shall study the golden rule for the transition probability per unit time:

$$
w_{i f}=\frac{2 \pi}{\hbar}\left|\left\langle f\left|H_{\mathrm{int}}^{\prime}\right| i\right\rangle+\sum_{I} \frac{\left\langle f\left|H_{\mathrm{int}}^{\prime}\right| I\right\rangle\left\langle I\left|H_{\mathrm{int}}^{\prime}\right| i\right\rangle}{E_{i}-E_{I}}\right|^{2} \delta\left(E_{f}-E_{i}\right),
$$

where $H_{\text {int }}^{\prime}$ is the time-independent part of the photonelectron interaction operator, $|i\rangle$ and $|f\rangle$ are the initial and the final states of the electron-photon system, and the transition $|i\rangle \rightarrow|f\rangle$ corresponds to the transition $|A\rangle \rightarrow|B\rangle$ between many-electron eigenstates of the system.

In the Dirac theory of the electron the total photonelectron interaction Hamiltonian is ${ }^{20}$ as

$$
\begin{aligned}
H_{\text {int }}(t) & =\int_{\infty} \mathcal{H}_{\text {int }}(\vec{r}, t) d^{3} r=\int_{\infty}-j_{\mu} A_{\mu} / c d^{3} r \\
& =-e \int_{\infty} \psi^{\dagger}(\vec{r}, t) \vec{\alpha} \psi(\vec{r}, t) \cdot \vec{A}(\vec{r}, t) d^{3} r,
\end{aligned}
$$

where the scalar potential part $\left(e A_{0}\right)$ of the interaction has been included to the nonperturbed Hamiltonian. The quantized radiation field operator $\vec{A}(\vec{r}, t)$ in Heaviside-Lorentz (rationalized) units can be written as

$$
\begin{aligned}
\vec{A}(\vec{r}, t)= & \sum_{\vec{q} \lambda}\left(\frac{\hbar c^{2}}{2 V \omega_{q}}\right)^{1 / 2} \\
& \times\left[a_{\vec{q} \lambda}(t) \hat{\boldsymbol{\epsilon}}^{(\lambda)} e^{i \vec{q} \cdot \vec{r}}+a_{\vec{q} \lambda}^{\dagger}(t) \hat{\boldsymbol{\epsilon}}^{(\lambda)^{*}} e^{-i \vec{q} \cdot \vec{r}}\right],
\end{aligned}
$$

where $a_{q \lambda}$ and $a_{\vec{q} \lambda}^{\dagger}$ are the photon annihilation and creation operators, respectively, and $\left\{\hat{\boldsymbol{\epsilon}}^{(\lambda)}\right\}$ is a set of unit, orthogonal, polarization vectors. The polarization index $\lambda=(+)$ for left and ( - ) for right circularly polarized photons, and $\hbar \vec{q}$ is the momentum of the photon. The normalization volume of the radiation field is denoted by $V$.

The quantized Dirac field operators $\psi$ and $\psi^{\dagger}$ in occupation number space can be written as

$$
\psi(\vec{r}, t)=\sum_{\Lambda} b_{\Lambda}(t) u_{\Lambda}(\vec{r})+d_{\Lambda}^{\dagger}(t) v_{\Lambda}(\vec{r})
$$

and

$$
\psi^{\dagger}(\vec{r}, t)=\sum_{\Lambda} b_{\Lambda}^{\dagger}(t) u_{\Lambda}^{\dagger}(\vec{r})+d_{\Lambda}(t) v_{\Lambda}^{\dagger}(\vec{r})
$$

where $u_{\Lambda}(\vec{r})$ and $v_{\Lambda}(\vec{r})$ are positive-energy electron and positron eigenstates of the Dirac Hamiltonian for the crystal, and form a complete orthonormal set of four component basis functions in the Dirac space. The (positive-energy) electron annihilation and creation operators are represented by $b_{\Lambda}$ and $b_{\Lambda}^{\dagger}$, respectively, and the corresponding operators for positrons are described by $d_{\Lambda}$ and $d_{\Lambda}^{\dagger} \cdot{ }^{23}$

In the case of anomalous (elastic, resonant) scattering, which we are interested in, the initial and final states in the electron-photon occupation number space can be written in the direct product form between many-electron and manyphoton states as

$$
\begin{aligned}
& |i\rangle=\left|\Psi_{0}\right\rangle \otimes\left|n_{\vec{q} \lambda}=1, n_{\vec{q}^{\prime} \lambda^{\prime}}=n_{\vec{q}^{\prime \prime} \lambda^{\prime \prime}}=\ldots=0\right\rangle, \\
& |f\rangle=\left|\Psi_{0}\right\rangle \otimes\left|n_{\overrightarrow{q^{\prime} \lambda^{\prime}}}=1, n_{\vec{q} \lambda}=n_{\vec{q}^{\prime \prime} \lambda^{\prime \prime}}=\ldots=0\right\rangle,
\end{aligned}
$$

where the electron system ground state $\left|\Psi_{0}\right\rangle$ in the Fock space reads as

$$
\begin{aligned}
\left|\Psi_{0}\right\rangle= & \mid n_{\Lambda_{i}}\left(\epsilon_{\Lambda_{i}}<0\right)=1 ; n_{\Lambda_{j}}\left(0<\epsilon_{\Lambda_{j}} \leqslant \epsilon_{F}\right)=1 ; \\
& \left.\times n_{\Lambda_{N+k}}\left(\epsilon_{\Lambda_{N+k}}>\epsilon_{F}\right)=0\right\rangle,
\end{aligned}
$$

where the single-particle state indices $i=\{-1,-2, \ldots,-\infty\}, \quad j=\{1,2, \ldots, N\}, \quad$ and $k=\{1,2, \ldots, \infty\}$ refer to the completely filled Dirac sea of negative-energy states, the filled positive-energy core and band states, and the empty states above the Fermi level, respectively. The labels $(\vec{q} \lambda)$ and $\left(\vec{q}^{\prime} \lambda^{\prime}\right)$ are related to the incoming and outgoing photons.

By virtue of the general many-photon state properties

$$
\begin{gathered}
a_{\vec{q} \lambda}\left|n_{\vec{q} \lambda}\right\rangle=\sqrt{n_{\vec{q} \lambda}}\left|n_{\vec{q} \lambda}-1\right\rangle, \\
a_{\vec{q} \lambda}^{\dagger}\left|n_{\vec{q} \lambda}\right\rangle=\sqrt{n_{\vec{q} \lambda}+1}\left|n_{\vec{q} \lambda}\right\rangle, \\
\left\langle n_{\vec{q} \lambda} \mid n_{\vec{q}^{\prime} \lambda^{\prime}}\right\rangle=\delta_{\vec{q} \vec{q}^{\prime}} \delta_{\lambda \lambda^{\prime}},
\end{gathered}
$$

and the fact that $H_{\text {int }}(t)$ is linear in the vector potential, the first-order term $\left\langle f\left|H_{\text {int }}^{\prime}\right| i\right\rangle$ in Eq. (1) vanishes identically. Therefore only the second-order term

$$
\begin{aligned}
\sum_{I} \frac{\left\langle f\left|H_{\mathrm{int}}^{\prime}\right| I\right\rangle\left\langle I\left|H_{\mathrm{int}}^{\prime}\right| i\right\rangle}{E_{i}-E_{I}}= & \sum_{I}\left\{\left\langle f\left|\int_{\infty} \psi^{\dagger}(\vec{r})[-e \vec{\alpha} \cdot \vec{A}(\vec{r})] \psi(\vec{r}) d^{3} r\right| I\right\rangle\right. \\
& \left.\times\left\langle I\left|\int_{\infty} \psi^{\dagger}\left(\vec{r}^{\prime}\right)\left[-e \vec{\alpha} \cdot \vec{A}\left(\vec{r}^{\prime}\right)\right] \psi\left(\vec{r}^{\prime}\right) d^{3} r^{\prime}\right| i\right\rangle\right\} /\left(E_{i}-E_{I}\right)
\end{aligned}
$$




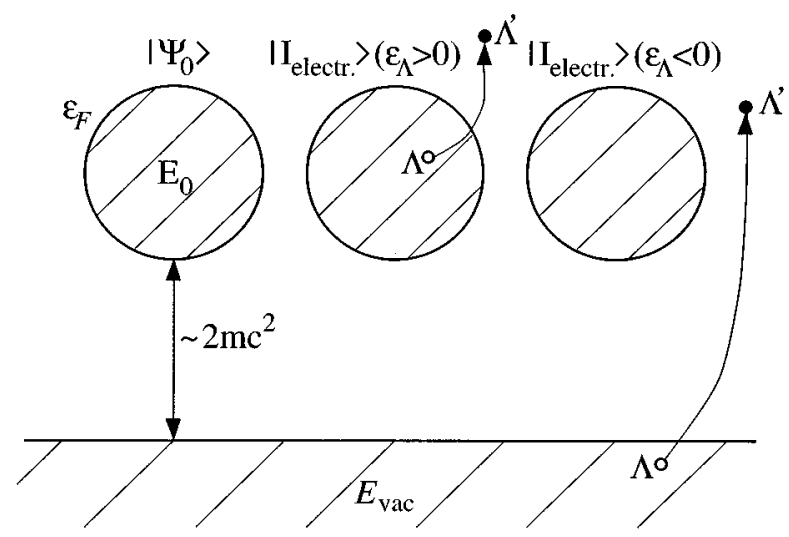

FIG. 1. A schematic description of the many-electron ground and intermediate (excited) states in the relativistic x-ray-scattering theory. $E_{0}$ and $E_{\mathrm{vac}}$ are the many-particle energies corresponding to the filled positive-energy states below the Fermi level $\epsilon_{F}$ and the filled negative-energy Dirac sea, respectively. The single-particle energies are denoted by $\epsilon_{\Lambda}$ and $\epsilon_{\Lambda^{\prime}}$.

contributes to the scattering amplitude. ${ }^{24}$ Index $I$ runs over the intermediate states, whose electron part $\left|I_{\text {elect }}\right\rangle$ contains excitations from the positive-energy as well as from the negative-energy states.

Note that there are just two types of intermediate states $|I\rangle$ involved in the scattering process: namely, those which have no photons and those having incoming and outgoing photons. The same situation occurs in the second-order term of the nonrelativistic scattering theory. ${ }^{3}$

The denominator of Eq. (7) can be written in terms of the eigenenergies $\epsilon_{\Lambda}$ of the effective one-particle Dirac equation (discussed later on) for the crystal and the incoming and outgoing photon energies $\hbar \omega_{q}$ and $\hbar \omega_{q^{\prime}}$ as

$$
\begin{aligned}
E_{i}-E_{I} & =\left(E_{\mathrm{vac}}+E_{0}+\hbar \omega_{q}\right)-\left(E_{\mathrm{vac}}+E_{0}-\epsilon_{\Lambda}+\epsilon_{\Lambda^{\prime}}\right) \\
& =\epsilon_{\Lambda}-\epsilon_{\Lambda^{\prime}}+\hbar \omega_{q}
\end{aligned}
$$

for the first term with a "no photon"-intermediate state and

$$
\begin{aligned}
E_{i}-E_{I}= & \left(E_{\mathrm{vac}}+E_{0}+\hbar \omega_{q}\right) \\
& -\left(E_{\mathrm{vac}}+E_{0}-\epsilon_{\Lambda}+\epsilon_{\Lambda^{\prime}}+\hbar \omega_{q}+\hbar \omega_{q^{\prime}}\right) \\
= & \epsilon_{\Lambda}-\epsilon_{\Lambda^{\prime}}-\hbar \omega_{q^{\prime}}
\end{aligned}
$$

for the second term with a "two photon"-intermediate state. In Eq. (8) $E_{0}$ and $E_{\text {vac }}$ are the energies of the many-particle systems, which correspond to the filled positive-energy states below the Fermi energy $\epsilon_{F}$ and the completely filled negative-energy sea (Dirac sea), respectively (see Fig. 1). The single-particle energies are subject to the constraints $\epsilon_{\Lambda} \leqslant \epsilon_{F}$ (either positive- or negative-energy state), and $\epsilon_{\Lambda^{\prime}}>\epsilon_{F}$. Figure 1 schematically describes the meaning of the various energy symbols in Eq. (8).

\section{B. Positive-energy part of the scattering amplitude}

We limit ourselves here to the case where the intermediate states $|I\rangle$ have excitations only from the positive-energy core or conduction band states $\left(\epsilon_{\Lambda}>0\right)$. Then, applying the time-independent expansions of the quantized vector potential $\vec{A}(\vec{r})$ [cf. Eq. (3)] and of the quantized Dirac field operators $\psi(\vec{r})$ and $\psi^{\dagger}(\vec{r})$ [cf. Eq. (4)] in Eq. (7), we find, in the case of elastic scattering, $\hbar \omega_{q}=\hbar \omega_{q^{\prime}} \equiv \hbar \omega$, for the positiveenergy part of the scattering amplitude ${ }^{25}$

$$
\begin{aligned}
\sum_{I, \epsilon_{\Lambda}>0} \frac{\left\langle f\left|H_{\mathrm{int}}^{\prime}\right| I\right\rangle\left\langle I\left|H_{\mathrm{int}}^{\prime}\right| i\right\rangle}{E_{i}-E_{I}}= & \sum_{\Lambda \Lambda^{\prime}} \int d^{3} r u_{\Lambda}^{\dagger}(\vec{r}) X_{q^{\prime} \lambda^{\prime}}^{\dagger}(\vec{r}) u_{\Lambda^{\prime}}(\vec{r}) \int d^{3} r^{\prime} u_{\Lambda^{\prime}}^{\dagger}\left(\vec{r}^{\prime}\right) X_{\vec{q} \lambda}\left(\vec{r}^{\prime}\right) u_{\Lambda}\left(\vec{r}^{\prime}\right) /\left(\epsilon_{\Lambda}-\epsilon_{\Lambda^{\prime}}+\hbar \omega\right) \\
& +\sum_{\Lambda \Lambda^{\prime}} \int d^{3} r u_{\Lambda}^{\dagger}(\vec{r}) X_{\vec{q} \lambda}(\vec{r}) u_{\Lambda^{\prime}}(\vec{r}) \int d^{3} r^{\prime} u_{\Lambda^{\prime}}^{\dagger}\left(\vec{r}^{\prime}\right) X_{q^{\prime} \lambda^{\prime}}^{\dagger}\left(\vec{r}^{\prime}\right) u_{\Lambda}\left(\vec{r}^{\prime}\right) /\left(\epsilon_{\Lambda}-\epsilon_{\Lambda^{\prime}}-\hbar \omega\right),
\end{aligned}
$$

where the relativistic photon-electron interaction vertex $X_{\vec{q} \lambda}(\vec{r})$ is defined by

$$
X_{\vec{q} \lambda}(\vec{r}) \equiv-e\left(\frac{\hbar c^{2}}{2 V \omega_{q}}\right)^{1 / 2} \vec{\alpha} \cdot \hat{\epsilon}^{(\lambda)} e^{i \vec{q} \cdot \vec{r}}
$$

and the one-electron state labels $\Lambda$ and $\Lambda^{\prime}$ are subject to the constraints $0<\epsilon_{\Lambda} \leqslant \epsilon_{F}$ and $\epsilon_{\Lambda^{\prime}}>\epsilon_{F}$.

We can cast Eq. (9) into an even more useful form by recalling the eigenfunction expansion of the Dirac Green's function and noting that

$$
\begin{aligned}
-\frac{1}{\pi} \operatorname{Im} \mathcal{G}\left(\vec{r}, \vec{r}^{\prime}, \epsilon\right)= & \sum_{\Lambda^{\prime}(\text { all })} u_{\Lambda^{\prime}}(\vec{r}) \delta\left(\epsilon-\epsilon_{\Lambda^{\prime}}^{u}\right) u_{\Lambda^{\prime}}^{\dagger}\left(\vec{r}^{\prime}\right) \\
& +v_{\bar{\Lambda}^{\prime}}(\vec{r}) \delta\left(\epsilon-\epsilon_{\bar{\Lambda}^{\prime}}^{v}\right) v_{\bar{\Lambda}^{\prime}}^{\dagger}\left(\vec{r}^{\prime}\right),
\end{aligned}
$$

where $\epsilon_{\Lambda^{\prime}}^{u}$ and $\epsilon_{\bar{\Lambda}}^{v}$, are positive- and negative-energy eigenvalues of the Dirac equation, respectively, and $v_{\bar{\Lambda}^{\prime}}(\vec{r})$ is the negative-energy electron state directly related to the corresponding positive-energy positron state $v_{\Lambda^{\prime}}(\vec{r})$. Then the expression for the first term of Eq. (9a), which is denoted as a 
scattering amplitude $f_{\vec{q} \lambda: q^{\prime} \lambda^{\prime}}^{+ \text {(pos) }}$, turns out to be

$$
\begin{aligned}
f_{\vec{q} \lambda ; \vec{q}^{\prime} \lambda^{\prime}}^{+(\text {pos })}(\omega)= & -\sum_{\Lambda} \int_{\infty} d^{3} r \int_{\infty} d^{3} r^{\prime} u_{\Lambda}^{\dagger}(\vec{r}) X_{q^{\prime} \lambda^{\prime}}^{\dagger}(\vec{r}) \\
& \times \int_{-\infty}^{\infty} \frac{d \epsilon}{\pi} \frac{\operatorname{Im} \mathcal{G}\left(\vec{r}, \vec{r}^{\prime}, \epsilon\right) \theta\left(\epsilon-\epsilon_{F}\right)}{\epsilon_{\Lambda}-\epsilon+\hbar \omega} \\
& \times X_{\vec{q} \lambda}\left(\vec{r}^{\prime}\right) u_{\Lambda}\left(\vec{r}^{\prime}\right),
\end{aligned}
$$

which causes the anomalous scattering, when the photon energy approaches the absorption edge of some atomic constituent defined by the core level $\epsilon_{\Lambda}$. Similarly, the second term of Eq. (9a), which we denote as a scattering amplitude $f_{\vec{q} \lambda ; q^{\prime} \lambda^{\prime}}^{- \text {(pos) }}$, can be written as

$$
\begin{aligned}
f_{\vec{q} \lambda ; \vec{q}^{\prime} \lambda^{\prime}}^{-(\operatorname{pos})}(\omega)= & -\sum_{\Lambda} \int_{\infty} d^{3} r \int_{\infty} d^{3} r^{\prime} u_{\Lambda}^{\dagger}(\vec{r}) X_{\vec{q} \lambda}(\vec{r}) \\
& \times \int_{-\infty}^{\infty} \frac{d \epsilon \operatorname{Im} \mathcal{G}\left(\vec{r}, \vec{r}^{\prime}, \epsilon\right) \theta\left(\epsilon-\epsilon_{F}\right)}{\epsilon_{\Lambda}-\epsilon-\hbar \omega} \\
& \times X_{\vec{q}^{\prime} \lambda^{\prime}}^{\dagger}\left(\vec{r}^{\prime}\right) u_{\Lambda}\left(\vec{r}^{\prime}\right),
\end{aligned}
$$

where $\theta$ is the standard unit-step function. It is noticeable that the amplitude $f_{\vec{q} \lambda ; q^{\prime} \lambda^{\prime}}^{- \text {(pos) }}(\omega)$ is a smooth function of $\omega$, and is therefore responsible for the nonresonant scattering.

In Eq. (11) the site-diagonal (SD) Green's function ${ }^{26}$ for an infinite array of nonoverlapping muffin-tin potentials can be written (cf. Faulkner and Stocks ${ }^{27}$ ) as

$$
\begin{aligned}
\mathcal{G}^{+}\left(\vec{r}, \vec{r}^{\prime}, \epsilon\right)= & \sum_{\Lambda \Lambda^{\prime}} Z_{\Lambda}\left(\vec{r}_{n}, \epsilon\right) \tau_{\Lambda \Lambda^{\prime}}^{n n} Z_{\Lambda^{\prime}}^{\dagger}\left(\vec{r}_{n}^{\prime}, \epsilon\right) \\
& -\sum_{\Lambda} Z_{\Lambda}\left(\vec{r}_{n}, \epsilon\right) J_{\Lambda}^{\dagger}\left(\vec{r}_{n}^{\prime}, \epsilon\right),
\end{aligned}
$$

where $\quad \vec{r}_{n} \equiv \vec{r}-\vec{R}_{n}, \quad \vec{r}_{n}^{\prime} \equiv \vec{r}^{\prime}-\vec{R}_{n}, \quad$ and $\quad Z_{\Lambda}\left(\vec{r}_{n}, \epsilon\right) \quad$ and $J_{\Lambda}\left(\vec{r}_{n}, \epsilon\right)$ are the regular and irregular solutions of the spinpolarized Kohn-Sham-Dirac equation, respectively, around the $n$th site muffin-tin potential. ${ }^{17,28}$

Because the spin polarization of the sample breaks the crystal symmetry we have to express the solution $Z_{\Lambda}\left(\vec{r}_{n}, \epsilon\right)$ [and similarly $\left.J_{\Lambda}\left(\vec{r}_{n}, \epsilon\right)\right]$ as a linear combination ${ }^{29}$ of the bispinors as

$$
Z_{\Lambda}\left(\vec{r}_{n}, \epsilon\right)=Z_{\kappa \mu}\left(\vec{r}_{n}, \epsilon\right)=\sum_{\kappa^{\prime}} Z_{\kappa^{\prime} \kappa}^{\mu}\left(\vec{r}_{n}, \epsilon\right),
$$

where

$$
Z_{\kappa^{\prime} \kappa}^{\mu}(\vec{r}, \epsilon) \equiv\left(\begin{array}{c}
g_{\kappa^{\prime} \kappa}^{\mu}(r, \epsilon) \chi_{\kappa^{\prime}}^{\mu}(\hat{r}) \\
i f_{\kappa^{\prime} \kappa}^{\mu}(r, \epsilon) \chi_{-\kappa^{\prime}}^{\mu}(\hat{r})
\end{array}\right),
$$

and $\chi_{\kappa}^{\mu}(\hat{r})$ is the spin angular function ${ }^{19}$ and the radial functions $g_{\kappa^{\prime} \kappa}^{\mu}(r, \epsilon)$ and $f_{\kappa^{\prime} \kappa}^{\mu}(r, \epsilon)$ satisfy a set of coupled radial Dirac equations. ${ }^{18}$

As a usual approximation, we limit the values of $\kappa^{\prime}$ in Eq. (13) to $\{\kappa,-\kappa-1\}$, so that all couplings to the partial waves whose orbital angular momentum value differs from $l$ are neglected. As a consequence, the infinite set of the coupled radial equations has been reduced to only two sets of four coupled equations. ${ }^{18,30}$

Furthermore, in our calculations we choose the effective scalar potential and the spin-only effective exchange fields to be

$$
\begin{aligned}
& V^{\text {eff }}(r)=\frac{1}{2}\left[V_{\uparrow}(r)+V_{\downarrow}(r)\right], \\
& B^{\text {eff }}(r)=\frac{1}{2}\left[V_{\uparrow}(r)-V_{\downarrow}(r)\right],
\end{aligned}
$$

where $V_{\uparrow}(r)$ and $V_{\downarrow}(r)$ are the spin-up and spin-down spherically symmetric muffin-tin potentials which, for simplicity, we take from the nonrelativistic self-consistent, spinpolarized electronic structure calculation of Moruzzi et al. ${ }^{31}$ for metals and of Johnson et al. ${ }^{32}$ for disordered alloys. This should be a good approximation for the potentials of metals and alloys composed of light atoms, like $\mathrm{Fe}$ and $\mathrm{Ni}^{17}$

\section{Negative-energy part of the scattering amplitude}

We turn to discuss some important peculiarities related to the negative-energy electron states in the scattering amplitude formula, Eq. (7). As is well known relativistic scattering of low-energy photons $\left(\hbar \omega \ll m c^{2}\right.$ ) by free electrons (Thomson scattering) will necessarily involve the negative-energy states. These must be taken into account in computing the scattering cross section if we are to obtain the correct (nonrelativistic) result. In fact, the contribution of the positiveenergy states to the scattering cross section is vanishingly small compared to the negative-energy one in Thomson scattering. ${ }^{20}$

Second, we may recall ${ }^{20}$ that the contribution to the Thomson scattering amplitude by the negative-energy states, as derived using the Dirac hole theory in connection with the usual perturbation theory, has a correct magnitude, but a wrong sign.

Guided by the experience in this "subtle sign problem" in context of the relativistic Thomson scattering, we derive the expression for the elastic-scattering amplitude due to virtual excitations from the filled negative-energy states $\left(\epsilon_{\Lambda}<0\right)$, i.e., from the Dirac sea into the empty conduction band states. Proceeding otherwise similarly as we did in the positive-energy electron excitation case, the negative-energy states contribution to the scattering amplitude turns out to be

$$
\begin{aligned}
-\sum_{I, \epsilon_{\Lambda}<0} \frac{\left\langle f\left|H_{\mathrm{int}}^{\prime}\right| I\right\rangle\left\langle I\left|H_{\mathrm{int}}^{\prime}\right| i\right\rangle}{E_{i}-E_{I}}= & -\sum_{\Lambda \Lambda^{\prime}} \int d^{3} r v_{\bar{\Lambda}}^{\dagger}(\vec{r}) X_{q^{\prime} \lambda^{\prime}}^{\dagger}(\vec{r}) u_{\Lambda^{\prime}}(\vec{r}) \int d^{3} r^{\prime} u_{\Lambda^{\prime}}^{\dagger}\left(\vec{r}^{\prime}\right) X_{\vec{q} \lambda}\left(\vec{r}^{\prime}\right) v_{\bar{\Lambda}}\left(\vec{r}^{\prime}\right) /\left(\epsilon_{\Lambda}-\epsilon_{\Lambda^{\prime}}+\hbar \omega\right) \\
& \left.-\sum_{\Lambda \Lambda^{\prime}} \int d^{3} r v_{\bar{\Lambda}}^{\dagger}(\vec{r}) X_{\vec{q} \lambda}(\vec{r}) u_{\Lambda^{\prime}}(\vec{r}) \int d^{3} r^{\prime} u_{\Lambda^{\prime}}^{\dagger}\left(\vec{r}^{\prime}\right) X_{q^{\prime} \lambda^{\prime}}^{\dagger}\left(\vec{r}^{\prime}\right) v_{\bar{\Lambda}} \bar{r}^{\prime}\right) /\left(\epsilon_{\Lambda^{\prime}}-\epsilon_{\Lambda^{\prime}}-\hbar \omega\right),
\end{aligned}
$$

where the first and second terms may be usefully identified as the scattering amplitudes $f_{\vec{q} \lambda ; q^{\prime} \lambda^{\prime}}^{+(\text {neg })}$ and $f_{\vec{q} \lambda \overrightarrow{q^{\prime}} \lambda^{\prime}}^{-(\text {neg) }}$, respectively. It can be directly noted from Eq. (15) that none of these terms have abrupt resonances in practical circumstances, since 
$\epsilon_{\Lambda}-\epsilon_{\Lambda^{\prime}}<-2 m c^{2}$ and $\hbar \omega \ll 2 m c^{2}$. Furthermore, these scattering amplitudes can be readily expressed in terms of the Green's function as

$$
f_{\overrightarrow{q \lambda} ; \overrightarrow{q^{\prime} \lambda^{\prime}}}^{+(\mathrm{neg})}(\omega)=\sum_{\Lambda} \int_{\infty} d^{3} r \int_{\infty} d^{3} r^{\prime} v_{\bar{\Lambda}}^{\dagger}(\vec{r}) X_{\vec{q}^{\prime} \lambda^{\prime}}^{\dagger}(\vec{r}) \int_{-\infty}^{\infty} \frac{d \epsilon}{\pi} \frac{\operatorname{Im} \mathcal{G}\left(\vec{r}, \vec{r}^{\prime}, \epsilon\right) \theta\left(\epsilon-\epsilon_{F}\right)}{\epsilon_{\Lambda}-\epsilon+\hbar \omega} X_{\vec{q} \lambda}\left(\vec{r}^{\prime}\right) v_{\bar{\Lambda}}\left(\vec{r}^{\prime}\right)
$$

and

$$
f_{\vec{q} \lambda ; \vec{q}^{\prime} \lambda^{\prime}}^{-(\operatorname{neg})}(\omega)=\sum_{\Lambda} \int_{\infty} d^{3} r \int_{\infty} d^{3} r^{\prime} v_{\bar{\Lambda}}^{\dagger}(\vec{r}) X_{q \lambda}(\vec{r}) \int_{-\infty}^{\infty} \frac{d \epsilon}{\pi} \frac{\operatorname{Im} \mathcal{G}\left(\vec{r}, \vec{r}^{\prime}, \epsilon\right) \theta\left(\epsilon-\epsilon_{F}\right)}{\epsilon_{\Lambda}-\epsilon-\hbar \omega} X_{\vec{q}^{\prime} \lambda^{\prime}}^{\dagger}\left(\vec{r}^{\prime}\right) v_{\bar{\Lambda}}\left(\vec{r}^{\prime}\right),
$$

where $\left\{\epsilon_{\Lambda}\right\}$ forms an infinite continuum of the negativeenergy electron states. We summarize our main results [Eqs. (11) and (16)] by writing the fully relativistic, elasticscattering amplitude as

$$
\begin{aligned}
f_{\vec{q} \lambda ; \vec{q}^{\prime} \lambda^{\prime}}(\omega)= & f_{\vec{q} \lambda ; \vec{q}^{\prime} \lambda^{\prime}}^{+(\operatorname{pos})}(\omega)+f_{\vec{q} \lambda ; q^{\prime} \lambda^{\prime}}^{-(\operatorname{pos})}(\omega) \\
& +f_{\vec{q} \lambda ; \vec{q}^{\prime} \lambda^{\prime}}^{+(\text {neg })}(\omega)+f_{\vec{q} \lambda ; \vec{q}^{\prime} \lambda^{\prime}}^{-(\text {neg })}(\omega),
\end{aligned}
$$

where the first term $f_{\vec{q} \lambda ; q^{\prime} \lambda^{\prime}}^{+ \text {(pos) }}(\omega)$ causes the resonant magnetic scattering, when $\hbar \omega \rightarrow \epsilon_{F}-\epsilon_{\Lambda}$, and the rest of the three terms are smooth functions of frequency. It is interesting to compare this with the corresponding nonrelativistic formula by Durham, ${ }^{21}$ which has been derived using the more sophisticated Keldysh-diagram technique. ${ }^{22}$ As expected, his result bears a close resemblance to our expression except that in the nonrelativistic theory just three terms appear, and no conceptually difficult "negative-energy" problem arises. In the nonrelativistic theory the scattering amplitude is obtained as a sum of three contributions:

$$
f(\omega)=f^{0}+f^{+}(\omega)+f^{-}(\omega),
$$

where $f^{0}$ represents the frequency-independent charge scattering, $f^{+}(\omega)$ is responsible for the anomalous scattering, and $f^{-}(\omega)$ is a smooth function of frequency. Furthermore, in the relativistic, Lorentz-invariant formula for the scattering amplitude, all terms depend explicitly on frequency, and there is no separate term for charge scattering. In fact, all possible scattering sources (charge scattering, spin scattering, etc.) are coupled together in an intricate way in various terms of Eq. (17).

In spite of the dramatic importance of the negative-energy states in Thomson scattering, we are going to ignore them, and only the first two terms of Eq. (17) will be retained. This procedure may be justified as follows. (i) Only the $f^{+(\operatorname{pos})}(\omega)$ term of Eq. (17) is responsible for the resonance, anomalous scattering behavior in practical conditions of $\hbar \omega \ll 2 m c^{2}$. (ii) Unlike in Thomson scattering, where all electron states (positive and negative energy) in the matrix elements of Eq. (7) are free-electron-like, the negativeenergy states in the solid state environment are largely extended while the states near the Fermi level are more localized. Thus, the corresponding matrix elements should be essentially smaller than in the case where both states in the matrix elements would be extended.

It would be an interesting task itself to evaluate quantitatively the negative-energy states contribution to the scatter- ing amplitude. However, this seems to be quite a difficult problem, because (i) unlike the core states, the negativeenergy states $\left\{v_{\bar{\Lambda}}(\vec{r})\right\}$ form a continuum spectrum $\left(\epsilon_{\Lambda}<-m c^{2}\right.$ ), and (ii) $\left\{v_{\bar{\Lambda}}(\vec{r})\right\}$ are extendedlike states, so that in addition to the site-diagonal (SD) Green's function also the non-site-diagonal (NSD) Green's function would be required for the computation.

\section{Magnetic Bragg scattering}

In order to show some realization of the general scattering formula, Eq. (17), we apply it to one of the simplest cases, namely, to magnetic Bragg scattering in a perfectly ordered infinite ferromagnet. In such a system the Green's function $\mathcal{G}\left(\vec{r}, \vec{r}^{\prime}, \epsilon\right)$ and the path operators $\tau^{n n}$ in Eq. (17) have the translational invariance of the lattice, i.e., $\mathcal{G}\left(\vec{r}+\vec{R}_{n}, \vec{r}^{\prime}+\vec{R}_{n}, \epsilon\right)=\mathcal{G}\left(\vec{r}, \vec{r}^{\prime}, \epsilon\right)$ and $\tau^{n n}=\tau^{00}$. Also, each site has an identical contribution to the total scattering amplitude. It will be then straightforward to show that the site decomposition of the Bragg scattering amplitude (due to positive-energy excitations only) becomes as

$$
\begin{aligned}
f^{(\operatorname{pos})}(\omega) & =\sum_{n}\left[f_{n}^{+(\operatorname{pos})}(\omega)+f_{n}^{-(\operatorname{pos})}(\omega)\right] e^{-i \vec{Q} \cdot \vec{R}_{n}} \\
& =\left[f_{0}^{+(\operatorname{pos})}(\omega)+f_{0}^{-(\operatorname{pos})}(\omega)\right] \sum_{n} e^{-i \vec{Q} \cdot \vec{R}_{n}} \\
& =\left[f_{0}^{+(\operatorname{pos})}(\omega)+f_{0}^{-(\operatorname{pos})}(\omega)\right] N_{\text {atoms }} \delta_{\vec{Q} \vec{K}}
\end{aligned}
$$

where $\vec{Q} \equiv \vec{q}^{\prime}-\vec{q}, \vec{K}$ is a reciprocal lattice vector of the ferromagnet, and $\delta_{O K}$ is a Kronecker delta function between $\vec{Q}$ and $\vec{K}$. The resonant part of the amplitude, i.e., $f_{0}^{+(\operatorname{pos})}(\omega)$, is given by

$$
f_{0}^{+(\operatorname{pos})}(\omega)=\sum_{\Lambda_{0}} f_{\Lambda_{0} ; \vec{q} \lambda ; \vec{q}^{\prime} \lambda^{\prime}}^{+(\omega)}(\omega)
$$

where the $\Lambda_{0}$-core-state contribution is

$$
\begin{aligned}
& f_{\Lambda_{0} ; \vec{q} \lambda ; q^{\prime} \lambda^{\prime}}^{+(\operatorname{pos})}(\omega)= \\
& \quad-\sum_{\Lambda \Lambda^{\prime}} \int_{\epsilon_{F}}^{\infty} \frac{d \epsilon}{\pi} \frac{m_{\Lambda_{0}}^{\Lambda+}\left(\vec{q}^{\prime} \lambda^{\prime} ; \epsilon\right) \operatorname{Im} \tau_{\Lambda \Lambda^{\prime}}^{00}(\epsilon) m_{\Lambda_{0}}^{\Lambda^{\prime}+*}(\vec{q} \lambda ; \epsilon)}{\epsilon_{\Lambda_{0}}-\epsilon+\hbar \omega+i \Gamma / 2},
\end{aligned}
$$

and the matrix element $m_{\Lambda_{0}}^{\Lambda+}(\vec{q} \lambda ; \epsilon)$ is defined by 


$$
m_{\Lambda_{0}}^{\Lambda+}(\vec{q} \lambda ; \epsilon) \equiv \int_{\vec{r}_{0} \in \Omega_{0}} u_{\Lambda_{0}}^{\dagger}\left(\vec{r}_{0}\right) X_{q_{\lambda}}^{\dagger}\left(\vec{r}_{0}\right) Z_{\Lambda}\left(\vec{r}_{0}, \epsilon\right) d^{3} r_{0},
$$

where $\vec{r}_{0} \equiv \vec{r}-\vec{R}_{0}$ is the vector inside the unit cell $\Omega_{0}$ at the origin.

Similarly, the nonresonant part of the amplitude, i.e., $f_{0}^{-(\operatorname{pos})}(\omega)$, is given by

$$
f_{0}^{-(\operatorname{pos})}(\omega)=\sum_{\Lambda_{0}} f_{\Lambda_{0} ; \vec{q} \lambda ; \vec{q}^{\prime} \lambda^{\prime}}^{-(\operatorname{pos})}(\omega),
$$

with the $\Lambda_{0}$-core-state contribution as

$$
\begin{aligned}
& f_{\Lambda_{0} ; \vec{q} \lambda ; \vec{q}^{\prime} \lambda^{\prime}}^{-(\omega)}(\omega) \\
& \quad=-\sum_{\Lambda \Lambda^{\prime}} \int_{\epsilon_{F}}^{\infty} \frac{d \epsilon}{\pi} \frac{m_{\Lambda_{0}}^{\Lambda-}\left(\vec{q}^{\prime} \lambda^{\prime} ; \epsilon\right) \operatorname{Im} \tau_{\Lambda \Lambda^{\prime}}^{00}(\epsilon) m_{\Lambda_{0}}^{\Lambda^{\prime}-*}(\vec{q} \lambda ; \epsilon)}{\epsilon_{\Lambda_{0}}-\epsilon-\hbar \omega},
\end{aligned}
$$

where the matrix element $m_{\Lambda_{0}}^{\Lambda-}(\vec{q} \lambda ; \epsilon)$ is defined by

$$
m_{\Lambda_{0}}^{\Lambda-}(\vec{q} \lambda ; \epsilon) \equiv \int_{\vec{r}_{0} \in \Omega_{0}} u_{\Lambda_{0}}^{\dagger}\left(\vec{r}_{0}\right) X_{q \lambda}\left(\vec{r}_{0}\right) Z_{\Lambda}\left(\vec{r}_{0}, \epsilon\right) d^{3} r_{0}
$$

It has to be noticed that the irregular parts of the Green's function have no contribution at all to the scattering amplitude if the energy integral above the Fermi energy is made up using real energy points. However, in our practical calculations we have included a small positive imaginary part $(0.01 \mathrm{Ry})$ to our energy points in order to smooth the features in the path operator and consequently reduce the computation time of the energy integral. But our experience shows that in this case the contribution of the irregular part of the $G$ function is still very small compared to that of the regular part. Therefore we have ignored it in Eqs. (20) and (21). In the resonant part of the scattering amplitude, Eq. (20b), we have introduced $\Gamma$, the only adjustable parameter in the theory, to represent the natural width of the intermediate states. It is noticeable that in the magnetic absorption theory by Ebert et al. ${ }^{30}$ only one type of a matrix element is required, while in the $\mathrm{x}$-ray magnetic-scattering theory we need two different types of matrix elements: one for the resonant amplitude and one for the nonresonant one. When the frequency of the incoming x-ray beam is tuned to near the absorption edge, usually only one localized core state, say, $u_{\Lambda_{c}}(\vec{r})$, contributes significantly to the elastic-scattering amplitude. Then the summation index in Eqs. (20a) and (21a) can be restricted to a single term $\Lambda_{0}=\Lambda_{c}$.
And, finally, we mention a few practical points about the matrix elements in Eqs. (20) and (21). As we show below it is straightforward to derive numerically tractable approximations and selection rules for these matrix elements due to the electric dipole $(E 1)$ or magnetic dipole and electric quadrupole $(M 1+E 2)$ contributions to the photon-electron interaction vertex $X_{q \lambda}(\vec{r})$.

\section{Matrix elements in electric dipole approximation}

In the electric dipole approximation $\left[e^{i q \cdot \vec{r}} \approx 1\right.$ in Eq. (9b)], the matrix element $m_{\Lambda_{0}}^{\Lambda+}(\vec{q} \lambda ; \epsilon)$ in the resonant part of the scattering amplitude can be written as

$$
\begin{aligned}
m a_{\Lambda_{0}}^{\Lambda+}(\vec{q} \lambda ; \epsilon)= & -i e\left(\frac{\hbar c^{2}}{2 V \omega_{q}}\right)^{1 / 2} \sum_{\kappa_{0}^{\prime} \kappa^{\prime}} \\
& \times\left\{\left[\int_{0}^{R_{\mathrm{WS}}} d r_{0} r_{0}^{2} g_{\kappa_{0}{ }^{\prime} \kappa_{0}}^{\mu_{0}} *\left(r_{0}\right) f_{\kappa^{\prime} \kappa}^{\mu}\left(r_{0}, \epsilon\right)\right]\right. \\
& \times A_{\kappa_{0}{ }^{\prime} \mu_{0} ;-\kappa^{\prime} \mu}^{(-\lambda)}(\hat{q}) \\
& -\left[\int_{0}^{{ }_{\mathrm{WS}}} d r_{0} r_{0}^{2} f_{\kappa_{0}{ }^{\prime} \kappa_{0}}^{\mu_{0}} *\left(r_{0}\right) g_{\kappa^{\prime} \kappa^{\prime}}^{\mu}\left(r_{0}, \epsilon\right)\right] \\
& \left.\times A_{-\kappa_{0}{ }^{\prime} \mu_{0} ; \kappa^{\prime} \mu}^{(-\lambda)}(\hat{q})\right\},
\end{aligned}
$$

in terms of the radial and angular integrals; $R_{\mathrm{WS}}$ is the Wiegner-Seitz radius and the angular integrals are defined by $^{29}$

$$
A_{\kappa \mu ; \kappa^{\prime} \mu^{\prime}}^{(\lambda)}(\hat{q}) \equiv \int \chi_{\kappa}^{\mu \dagger}(\hat{r}) \vec{\sigma} \cdot \hat{\boldsymbol{\epsilon}}^{(\lambda)} \chi_{\kappa^{\prime}}^{\mu^{\prime}}(\hat{r}) d \Omega .
$$

The matrix elements $m a_{\Lambda_{0}}^{\Lambda-}(\vec{q} \lambda ; \epsilon)$ can readily be evaluated by making a replacement $(-\lambda) \rightarrow(\lambda)$ in the angular matrix elements of the right side of Eq. (22a).

In deriving Eq. (22) we have used the fact that $\hat{\epsilon}^{(\lambda)^{*}}=\hat{\epsilon}^{(-\lambda)}$ for circularly polarized light. If the photon propagates along the $z$ axis (direction of the magnetization), then the unit polarization vectors for the right (RCP) and left (LCP) circularly polarized photons are $\hat{\epsilon}_{z}^{(-)}=$ $(1 / \sqrt{2})(1,-i, 0)$ and $\hat{\epsilon}_{z}^{(+)}=(1 / \sqrt{2})(1, i, 0)$, respectively. For any other propagation direction $\hat{q}=\hat{q}\left(\theta_{q}, \phi_{q}\right)$ off from the $z$ axis the polarization vectors $\hat{\boldsymbol{\epsilon}}^{(-)}$and $\hat{\boldsymbol{\epsilon}}^{(+)}$are given by the rotation matrix $\mathbf{R}\left(\theta_{q}, \phi_{q}\right)$ applied on $\hat{\boldsymbol{\epsilon}}_{z}^{(-)}$and $\hat{\boldsymbol{\epsilon}}_{z}^{(+)}$, correspondingly. Using the orthonormality of the spherical harmonics $Y_{l}^{m}(\hat{r})$ the angular integrals of Eq. (22) become of the form

$$
\begin{aligned}
A_{\kappa \mu ; \kappa^{\prime} \mu^{\prime}}^{(\lambda)}(\hat{q})= & f_{11}\left(\theta_{q}, \phi_{q}, \lambda\right) C\left(l \frac{1}{2} j ; \mu-\frac{1}{2}, \frac{1}{2}\right) C\left(l^{\prime} \frac{1}{2} j^{\prime} ; \mu^{\prime}-\frac{1}{2}, \frac{1}{2}\right) \delta_{l l^{\prime}} \delta_{\mu \mu^{\prime}}+f_{12}\left(\theta_{q}, \phi_{q}, \lambda\right) C\left(l \frac{1}{2} j ; \mu-\frac{1}{2}, \frac{1}{2}\right) \\
& \times C\left(l^{\prime} \frac{1}{2} j^{\prime} ; \mu^{\prime}+\frac{1}{2},-\frac{1}{2}\right) \delta_{l l^{\prime}} \delta_{\mu \mu^{\prime}+1}+f_{21}\left(\theta_{q}, \phi_{q}, \lambda\right) C\left(l \frac{1}{2} j ; \mu+\frac{1}{2},-\frac{1}{2}\right) C\left(l^{\prime} \frac{1}{2} j^{\prime} ; \mu^{\prime}-\frac{1}{2}, \frac{1}{2}\right) \delta_{l l^{\prime}} \delta_{\mu \mu^{\prime}-1} \\
& +f_{22}\left(\theta_{q}, \phi_{q}, \lambda\right) C\left(l \frac{1}{2} j ; \mu+\frac{1}{2},-\frac{1}{2}\right) C\left(l^{\prime} \frac{1}{2} j^{\prime} ; \mu^{\prime}+\frac{1}{2},-\frac{1}{2}\right) \delta_{l l^{\prime}} \delta_{\mu \mu^{\prime}},
\end{aligned}
$$


where the elements $f_{i j}\left(\theta_{q}, \phi_{q}, \lambda\right) \equiv\left(\vec{\sigma} \cdot \hat{\boldsymbol{\epsilon}}^{(\lambda)}\right)_{i j}$. For example, if the direction of $\vec{q}$ is described by a rotation around the $y$ axis by $\theta_{q}$ off from the $z$ axis $\left(\phi_{q}=0\right)$, then

$$
\left(\begin{array}{ll}
f_{11}\left(\theta_{q},+\right) & f_{12}\left(\theta_{q},+\right) \\
f_{21}\left(\theta_{q},+\right) & f_{22}\left(\theta_{q},+\right)
\end{array}\right)=\left(\begin{array}{cc}
-\sin \theta_{q} & 1+\cos \theta_{q} \\
\cos \theta_{q}-1 & \sin \theta_{q}
\end{array}\right)
$$

for the $\mathrm{LCP}$ radiation and

$$
\left(\begin{array}{ll}
f_{11}\left(\theta_{q},-\right) & f_{12}\left(\theta_{q},-\right) \\
f_{21}\left(\theta_{q},-\right) & f_{22}\left(\theta_{q},-\right)
\end{array}\right)=\left(\begin{array}{cc}
-\sin \theta_{q} & \cos \theta_{q}-1 \\
1+\cos \theta_{q} & \sin \theta_{q}
\end{array}\right)
$$

for the RCP radiation. The angular matrix elements of Eq. (23) solely determine the selection rules in the electric dipole approximation. Clearly $l-l_{0}= \pm 1$ for RCP and for LCP radiation in any propagation direction, while $\mu-\mu_{0}=0, \pm 1$, depending on the polarization state as well as on the propagation direction of the photon. It is also noticeable that the selection rules for the matrix elements $m a_{\Lambda_{0}}^{\Lambda+}(\vec{q} \lambda ; \epsilon)$ and $m a_{\Lambda_{0}}^{\Lambda-}(\vec{q} \lambda ; \epsilon)$ are slightly different with respect to the azimuthal $\mu$ quantum number, because Eq. (22a) contains an angular integral of the form $A^{(-\lambda)}$, while the expression for $m a_{\Lambda_{0}}^{\Lambda-}(\vec{q} \lambda ; \epsilon)$ contains $A^{(\lambda)}$ with an opposite polarization state index. These facts can be directly exploited in designing experimental setups in a magnetic x-ray-scattering measurement.

\section{Matrix elements due to magnetic dipole and electric quadrupole correction}

We derive a general angle-dependent expression for the combined magnetic dipole and electric quadrupole $(M 1+E 2)$ correction to the the electric dipole approximation (E1) of the matrix elements of Eqs. (20c) and (21c). If we now approximate $e^{i \vec{q} \cdot \vec{r}} \approx 1+i \vec{q} \cdot \vec{r}$ in Eq. (9b) for $X_{q \lambda}(\vec{r})$, then the term $i \vec{q} \cdot \vec{r}$ is responsible for the $(M 1+E 2)$ corrections to the electric dipole approximated matrix elements $m a_{\Lambda_{0}+}^{{ }^{+}}(\vec{q} \lambda ; \epsilon)$ and $m a_{\Lambda_{0}}^{\Lambda-}(\vec{q} \lambda ; \epsilon)$, which we denote as $m b_{\Lambda_{0}^{+}}^{\Lambda^{+}}(\vec{q} \lambda ; \epsilon)$ and $m b_{\Lambda_{0}}^{\Lambda_{-}^{-}}(\vec{q} \lambda ; \epsilon)$, respectively.

It is then a straightforward matter to show that the matrix element $m b_{\Lambda_{0}}^{\Lambda^{+}}(\vec{q} \lambda ; \epsilon)$, related to the resonant part of the scattering amplitude, can be written as

$$
\begin{aligned}
m b_{\Lambda_{0}}^{\Lambda+}(\vec{q} \lambda ; \epsilon)= & -e\left(\frac{\hbar c^{2}}{2 V \omega_{q}}\right)^{1 / 2} q \sum_{\kappa_{0}^{\prime} \kappa^{\prime}}\left\{\left[\int_{0}^{R_{\mathrm{WS}}} d r_{0} r_{0}{ }^{3} g_{\kappa_{0}^{\prime} \kappa_{0}}^{\mu_{0}} *\left(r_{0}, \epsilon_{\Lambda_{0}}\right) f_{\kappa^{\prime} \kappa^{\prime}}^{\mu}\left(r_{0}, \epsilon\right)\right] B_{\kappa_{0} \mu_{0} ;-\kappa^{\prime} \mu}^{(-\lambda)}(\hat{q})\right. \\
& \left.-\left[\int_{0}^{R_{\mathrm{WS}}} d r_{0} r_{0}{ }^{3} f_{\kappa_{0}{ }^{\prime} \kappa_{0}}^{\mu_{0}} *\left(r_{0}, \epsilon_{\Lambda_{0}}\right) g_{\kappa^{\prime} \kappa^{\prime}}^{\mu}\left(r_{0}, \epsilon\right)\right] B_{-\kappa_{0}{ }^{\prime} \mu_{0} ; \kappa^{\prime} \mu}^{(-\lambda)}(\hat{q})\right\},
\end{aligned}
$$

where the angular integrals are defined by

$$
B_{\kappa \mu ; \kappa^{\prime} \mu^{\prime}}^{(\lambda)}(\hat{q}) \equiv \int \chi_{\kappa}^{\mu \dagger}(\hat{r}) \vec{\sigma} \cdot \hat{\epsilon}^{(\lambda)} \hat{q} \cdot \hat{r} \chi_{\kappa^{\prime}}^{\mu^{\prime}}(\hat{r}) d \Omega
$$

where $|\hat{q}|=|\hat{r}|=1$.

A similar expression can be written for the nonresonant part of the scattering amplitude related matrix element $m b_{\Lambda_{0}}^{\Lambda-}(\vec{q} \lambda ; \epsilon)$ if we make the replacement $B^{(-\lambda)}(\hat{q})$ elements $\rightarrow-B^{(\lambda)}(\hat{q})$ elements on the right side of Eq. (25a).

If we assume a most general photon propagation direction $\hat{q}=\hat{q}\left(\theta_{q}, \phi_{q}\right)$ in terms of the standard polar $\left(\theta_{q}\right)$ and azimuthal $\left(\theta_{q}\right)$ angles, then it is a straightforward but lengthy matter to show that the angular matrix elements of Eq. (25b) can be written in the form

$$
\begin{aligned}
B_{\kappa \mu ; \kappa^{\prime} \mu^{\prime}}^{(\lambda)}(\hat{q})= & \sqrt{\frac{3\left(2 l^{\prime}+1\right)}{4 \pi(2 l+1)}} C\left(l^{\prime} 1 l ; 000\right) \delta_{\left|l-l^{\prime}\right|, 1(0)} u\left(l^{\prime}, 1, l\right)\left\{\delta _ { \mu - \mu ^ { \prime } , 0 } \left[C_{10} K_{11} C\left(l^{\prime} 1 l ; \mu^{\prime}-\frac{1}{2}, 0, \mu-\frac{1}{2}\right)\right.\right. \\
& \left.+C_{1-1} K_{12} C\left(l^{\prime} 1 l ; \mu^{\prime}+\frac{1}{2},-1, \mu-\frac{1}{2}\right)+C_{11} K_{21} C\left(l^{\prime} 1 l ; \mu^{\prime}-\frac{1}{2},+1, \mu+\frac{1}{2}\right)+C_{10} K_{22} C\left(l^{\prime} 1 l ; \mu^{\prime}+\frac{1}{2}, 0, \mu+\frac{1}{2}\right)\right] \\
& +\delta_{\mu-\mu^{\prime},-1}\left[C_{1-1} K_{11} C\left(l^{\prime} 1 l ; \mu^{\prime}-\frac{1}{2},-1, \mu-\frac{1}{2}\right)+C_{10} K_{21} C\left(l^{\prime} 1 l ; \mu^{\prime}-\frac{1}{2}, 0, \mu+\frac{1}{2}\right)\right. \\
& \left.+C_{1-1} K_{22} C\left(l^{\prime} 1 l ; \mu^{\prime}+\frac{1}{2},-1, \mu+\frac{1}{2}\right)\right]+\delta_{\mu-\mu^{\prime},+1}\left[C_{11} K_{11} C\left(l^{\prime} 1 l ; \mu^{\prime}-\frac{1}{2},+1, \mu-\frac{1}{2}\right)\right. \\
& \left.+C_{10} K_{12} C\left(l^{\prime} 1 l ; \mu^{\prime}+\frac{1}{2}, 0, \mu-\frac{1}{2}\right)+C_{11} K_{22} C\left(l^{\prime} 1 l ; \mu^{\prime}+\frac{1}{2},+1, \mu+\frac{1}{2}\right)\right] \\
& \left.+\delta_{\mu-\mu^{\prime},-2}\left[C_{1-1} K_{21} C\left(l^{\prime} 1 l ; \mu^{\prime}-\frac{1}{2},-1, \mu+\frac{1}{2}\right)\right]+\delta_{\mu-\mu^{\prime},+2}\left[C_{11} K_{12} C\left(l^{\prime} 1 l ; \mu^{\prime}+\frac{1}{2},+1, \mu-\frac{1}{2}\right)\right]\right\},
\end{aligned}
$$


where the photon propagation direction related coefficients $C_{1-1}, C_{10}$, and $C_{11}$ are given by

$$
\begin{gathered}
C_{1-1}\left(\theta_{q}, \phi_{q}\right)=\sqrt{\frac{2 \pi}{3}} \sin \theta_{q}\left(\cos \phi_{q}+i \sin \phi_{q}\right), \\
C_{10}\left(\theta_{q}\right)=\sqrt{\frac{4 \pi}{3}} \cos \theta_{q}, \\
C_{11}\left(\theta_{q}, \phi_{q}\right)=-\sqrt{\frac{2 \pi}{3}} \sin \theta_{q}\left(\cos \phi_{q}-i \sin \phi_{q}\right),
\end{gathered}
$$

the $K_{i j}$ elements are described in terms of the elements $f_{i j}=f_{i j}\left(\theta_{q}, \phi_{q}, \lambda\right)$ defined in Sec. II D 1 and the ClebschGordan coefficients ${ }^{33} C\left(l \frac{1}{2} j ; \mu-m_{s}, m_{s}\right)$ as

$$
\begin{gathered}
K_{11}=f_{11} C\left(l \frac{1}{2} j ; \mu-\frac{1}{2}, \frac{1}{2}\right) C\left(l^{\prime} \frac{1}{2} j^{\prime} ; \mu^{\prime}-\frac{1}{2}, \frac{1}{2}\right), \\
K_{12}=f_{12} C\left(l \frac{1}{2} j ; \mu-\frac{1}{2}, \frac{1}{2}\right) C\left(l^{\prime} \frac{1}{2} j^{\prime} ; \mu^{\prime}+\frac{1}{2},-\frac{1}{2}\right), \\
K_{21}=f_{21} C\left(l \frac{1}{2} j ; \mu+\frac{1}{2},-\frac{1}{2}\right) C\left(l^{\prime} \frac{1}{2} j^{\prime} ; \mu^{\prime}-\frac{1}{2}, \frac{1}{2}\right), \\
K_{22}=f_{22} C\left(l \frac{1}{2} j ; \mu+\frac{1}{2},-\frac{1}{2}\right) C\left(l^{\prime} \frac{1}{2} j^{\prime} ; \mu^{\prime}+\frac{1}{2},-\frac{1}{2}\right),
\end{gathered}
$$

and the function $u\left(l^{\prime}, 1, l\right)$ is defined by

$$
u\left(l^{\prime}, 1, l\right)=\left\{\begin{array}{lc}
0 & \text { if } l^{\prime}=l \\
1 & \text { otherwise }
\end{array}\right.
$$

In Eq. (26a) the coefficients $C\left(l^{\prime} 1 l ; \mu^{\prime}-m_{s}^{\prime}, m_{l_{2}}, \mu-m_{s}\right)$ are the usual Clebsch-Gordan coefficients $C\left(l_{1} l_{2} l ; m_{1}, m_{2}, m\right)$ with the angular momentum quantum number $l_{2}=1$.

Looking at Eq. (26) in the context of expression (25a) for the matrix element $m b_{\Lambda_{0}}^{\Lambda+}(\vec{q} \lambda ; \epsilon)$ [or in a context of a similar expression for $\left.m b_{\Lambda_{0}}^{\Lambda-}(\vec{q} \lambda ; \epsilon)\right]$, we notice that the selection rules of the $(M 1+E 2)$ contribution to the scattering can be determined in terms of the various Kronecker $\delta$ functions, the $u$ function, the angle-dependent coefficients $\left\{C_{l m}\right\}$, and the angle- and polarization-dependent elements $\left\{K_{i j}\right\}$. The resulting selection rules are then $l-l_{0}=0, \pm 1, \pm 2$ with the restriction that $s \rightarrow p$ and $p \rightarrow s$ be forbidden transitions, and for the azimuthal quantum number $\mu-\mu_{0}=0, \pm 1, \pm 2$ depending on the direction and polarization of the photon involved in the matrix element.

\section{E. Dichroism in magnetic x-ray scattering}

Our purpose here is to define what may become a useful observable in the state-of-the-art x-ray studies of magnetic materials, namely, the magnetic x-ray circular dichroism (MXCD) in context of magnetic $\mathrm{x}$-ray-scattering experiments. In a most general sense the dichroism (often called asymmetry ratio) in the elastic magnetic scattering of $\mathrm{x}$ rays can be defined in terms of the parameters $\left(\vec{q}, \vec{q}^{\prime}, \lambda, \lambda^{\prime}, \omega\right)$ of the "full experiment" as

$$
A\left(\vec{q} ; \vec{q}^{\prime} \lambda^{\prime} ; \omega\right) \equiv \frac{\omega_{d \Omega}\left(\vec{q}+; \vec{q}^{\prime} \lambda^{\prime} ; \omega\right)-\omega_{d \Omega}\left(\vec{q}-; \vec{q}^{\prime} \lambda^{\prime} ; \omega\right)}{\omega_{d \Omega}\left(\vec{q}+; \vec{q}^{\prime} \lambda^{\prime} ; \omega\right)+\omega_{d \Omega}\left(\vec{q}-; \vec{q}^{\prime} \lambda^{\prime} ; \omega\right)},
$$

where the scattering rate $\omega_{d \Omega}$ into a solid space angle $d \Omega$ can be written in terms of the scattering amplitude $f_{\vec{q} \lambda ; \vec{q}^{\prime} \lambda^{\prime}}\left(\omega, \omega^{\prime}\right)$ and the photon density of states function $\rho_{d \Omega}\left(\hbar \omega^{\prime}\right)$ for the emitted photons as ${ }^{20,3}$

$$
\begin{aligned}
\omega_{d \Omega} & =\int \frac{2 \pi}{\hbar}\left|f_{\vec{q} \lambda ; \vec{q}^{\prime} \lambda^{\prime}}\left(\omega, \omega^{\prime}\right)\right|^{2} \delta\left(E_{f}-E_{i}\right) \rho_{d \Omega}\left(\hbar \omega^{\prime}\right) d\left(\hbar \omega^{\prime}\right) \\
& =\frac{2 \pi}{\hbar}\left|f_{\vec{q} \lambda ; \vec{q}^{\prime} \lambda^{\prime}}(\omega)\right|^{2} \frac{V}{(2 \pi)^{3}} \frac{\omega^{2}}{\hbar c^{3}} d \Omega,
\end{aligned}
$$

where $\delta\left(E_{f}-E_{i}\right)=\delta\left(\hbar \omega^{\prime}-\hbar \omega\right)$ in the case of elastic scattering. An expression for the differential scattering cross section $d \delta / d \Omega$ results (see Sec. III A) if $\omega_{d \Omega} / d \Omega$ is divided by the incoming photon flux density $C / V$.

However, in the usual synchrotron experiment conditions it is very hard to measure the photon polarization state $\left(\lambda^{\prime}\right)$ of the weak emitted radiation. Therefore, the standard practice is to measure only the incoming beam polarization $(\lambda= \pm)$, and then to make a summation over the final state polarization index $\lambda^{\prime}$. Accordingly, the magnetic x-rayscattering dichroism (asymmetry ratio) used in our calculations will be defined as

$$
A\left(\vec{q}, \vec{q}^{\prime}, \omega\right) \equiv \frac{\sum_{\lambda^{\prime}}\left\{\left|f_{\vec{q}+; \vec{q}^{\prime} \lambda^{\prime}}(\omega)\right|^{2}-\left|f_{\vec{q}-; \vec{q}^{\prime} \lambda^{\prime}}(\omega)\right|^{2}\right\}}{\sum_{\lambda^{\prime}}\left\{\left|f_{\vec{q}+; \vec{q}^{\prime} \lambda^{\prime}}(\omega)\right|^{2}+\left|f_{\vec{q}-; \vec{q}^{\prime} \lambda^{\prime}}(\omega)\right|^{2}\right\}} .
$$

\section{RESULTS AND DISCUSSION}

We discuss the application of the theory to the resonant magnetic x-ray scattering at $L_{\mathrm{II}}, L_{\mathrm{III}}$, and $K$ absorption edges of ferromagnetically ordered iron. Although $\Gamma$ in Eq. (20b) is in general energy dependent, it is obviously a smooth function of energy, and therefore can be set as a good approximation to a constant value. In the case of the $K$-and $L_{\text {II,III-edge resonant scattering the atomic core-hole lifetime }}$ estimates $0.8 \mathrm{eV}$ (Ref. 34) and $1.0 \mathrm{eV}$ (Ref. 35) are used for $\Gamma$, respectively. ${ }^{36}$

The integration in Eq. (20b) has been performed using the energy range $\left[\epsilon_{F}, \epsilon_{F}+40 \mathrm{eV}\right]$, which we found to be sufficient to correctly produce the spectral features of interest. Even though it seems to be necessary to go beyond $100 \mathrm{eV}$ above the Fermi level in order to achieve a full convergence, we have noted that the spectral features do not change while moving the upper limit of the integral upwards from $40 \mathrm{eV}$ above the Fermi energy. Increasing the upper integration limit from 40 to $100 \mathrm{eV}$ simply increases the uniform background count.

\section{A. Magnetic scattering at $L_{\mathrm{II}}$ and $L_{\mathrm{III}}$ edges}

In Fig. 2 we present the calculated anomalous scattering cross section $d \sigma / d \Omega=|f(\omega)|^{2} V^{2} \omega^{2} /\left(2 \pi \hbar c^{2}\right)^{2}$ at the iron $L_{\text {II }}$ and $L_{\text {III }}$ absorption edges for right- and left-handedly polarized photons propagating along the magnetization direction. 


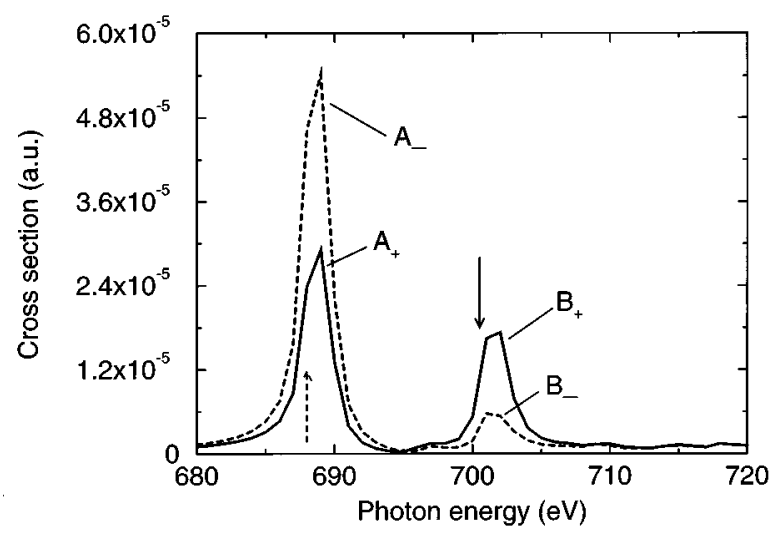

FIG. 2. Calculated anomalous scattering cross sections at the iron $L_{\mathrm{II}}$ and $L_{\mathrm{III}}$ absorption edges. The scattering amplitudes have been obtained by summing up the $L_{\mathrm{II}}$ and $L_{\text {III }}$ core state multiplet contributions simultaneusly. The solid and dashed lines correspond to left-handedly $(+)$ and right-handedly $(-)$ polarized incoming photons, respectively. In both cases the incoming and scattered photons propagate along the magnetization axis. The solid- and dashedline arrows locate the positions of the $L_{\mathrm{II}}$ and $L_{\mathrm{III}}$ edges, respectively, and the symbols $A_{+}, A_{-}$and $B_{+}, B_{-}$are related to $L_{\mathrm{III}^{-}}$and $L_{\mathrm{II}}$-edge resonant-scattering-derived peaks, respectively.

Interestingly, most features of interest in Fig. 2 can be qualitatively explained by looking numerically at the numerator part of Eq. (20b) just above the Fermi level, where the $d$ density of states contains a very strong and narrow peak. Our experience shows that it is exactly this peak in the unoccupied part of the iron band structure which is responsible for the overall behavior of the various resonant peaks $A_{+}, A_{-}, B_{+}$, and $B_{-}$in the spectra.

First of all we notice there are two major channels [cf. the numerator part of Eq. (20b)] for the $L_{\mathrm{II}}$-edge resonant scattering, while in $L_{\mathrm{III}}$ case there are several more, all of which are of similar order of magnitude. This explains, in general, why the peaks $A_{+}$and $A_{-}$are larger than $\mathrm{B}_{+}$and $\mathrm{B}_{-}$in Fig. 2. The specific character of the transition matrix elements involved in these channels depends on the selection rules together with the symmetry of the $\tau^{00}$ matrix. The selection rules for our x-ray-beam geometry are in $E 1$ approximation simply $l_{0}-l= \pm 1, \quad \mu=\mu_{0}+1$ for LCP radiation, and $\mu=\mu_{0}-1$ for RCP radiation. To be specific, we note that the transitions $\left(p_{1 / 2},-1 / 2\right) \rightarrow\left(d_{3 / 2},+1 / 2\right)$ and $\left(p_{1 / 2},+1 / 2\right) \rightarrow\left(d_{3 / 2},+3 / 2\right)$ mainly build up the $L_{\mathrm{II}}$-edge resonant scattering peak $B_{+}$for LCP radiation and the transitions $\quad\left(p_{1 / 2},-1 / 2\right) \rightarrow\left(d_{3 / 2},-3 / 2\right) \quad$ and $\quad\left(p_{1 / 2},+1 / 2\right)$ $\rightarrow\left(d_{3 / 2},-1 / 2\right)$ mainly contribute to peak $B_{-}$for RCP radiation.

On the other hand the $L_{\mathrm{III}}$-edge resonant peak does not possess a well-defined spin angular momentum character, because both $d_{3 / 2^{-}}$and $d_{5 / 2}$-like intermediate states participate with nearly equal weights in its intensity.

We also notice that the matrix elements of the transitions $\left(p_{3 / 2},-3 / 2\right) \rightarrow\left(d_{5 / 2},-5 / 2\right),\left(p_{3 / 2},-1 / 2\right) \rightarrow\left(d_{5 / 2},-3 / 2\right)$, and $\left(p_{3 / 2},+1 / 2\right) \rightarrow\left(d_{5 / 2},-1 / 2\right)$, which are related to $L_{\mathrm{III}}$-edge resonant peak for RCP radiation (peak $A_{-}$), are of similar size as those two involved in the $L_{\mathrm{II}}$-edge resonant peak (peak $B_{-}$). Therefore the $\tau^{00}$ matrix elements which are coupled to these transitions, and are essentially larger in the

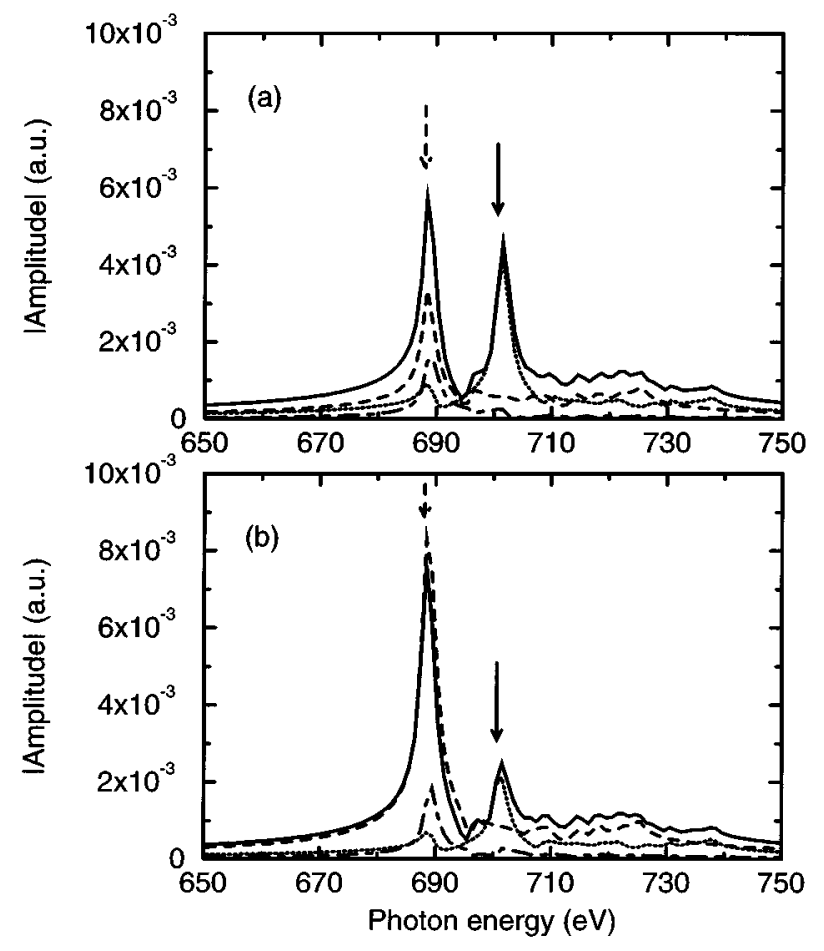

FIG. 3. Partial wave contributions to the scattering amplitude at the iron $L_{\mathrm{II}}$ and $L_{\mathrm{III}}$ absorption edges. The $L_{\mathrm{II}}$ and $L_{\mathrm{III}}$ core state multiplet contributions have been summed up simultaneusly. The solid, dotted, dashed, and dash-dotted lines correspond to total, $d_{3 / 2}, d_{5 / 2}$, and $d_{3 / 2}, d_{5 / 2}$ partial contributions, respectively. In (a) and (b) the incoming photons are left-handedly $(+)$ and righthandedly $(-)$ polarized, respectively. The photon beam geometry and the arrow symbols are the same as in Fig. 2.

case of the peak $A_{-}$than in the case of the peak $B_{-}$, clearly explain the large intensity difference between these two peaks in Fig. 2.

The above observations are well supported by the various partial wave contributions to the scattering amplitude ${ }^{25}$ presented in Fig. 3. There are two interesting points to note. First, the $\left(d_{3 / 2}, d_{5 / 2}\right)$-like spin-flip elements of the $\tau^{00}$ matrix, which reflect the crystal field effects, carry essential weight in the $L_{\mathrm{III}}$-derived resonant feature of Fig. 3. Second, the total scattering amplitude for the RCP scattering at the $L_{\text {III }}$ edge is slightly smaller than the $d_{5 / 2}$ contribution to the amplitude. This is due to the fact that the phase of the $\left(d_{3 / 2}, d_{5 / 2}\right)$ partial amplitude has been shifted by nearly $180^{\circ}$ with respect to the phases of the $d_{3 / 2^{-}}$and $d_{5 / 2}$-related amplitudes of Fig. 4(b).

\section{B. Magnetic scattering at the $K$ edge}

In Fig. 5 we present the calculated scattering cross section $d \sigma / d \Omega=|f(\omega)|^{2} V^{2} \omega^{2} /\left(2 \pi \hbar c^{2}\right)^{2}$ at the iron $K$ absorption edge for right-handedly polarized photons propagating along the magnetization direction. In order to demonstrate the remarkable dependence of the scattering at the $K$ edge on the crystal environment effects, we have shown in Fig. 5 the single-site scattering contribution to the cross section as well as the squared value of the $p$-projected density of states above the Fermi level. 


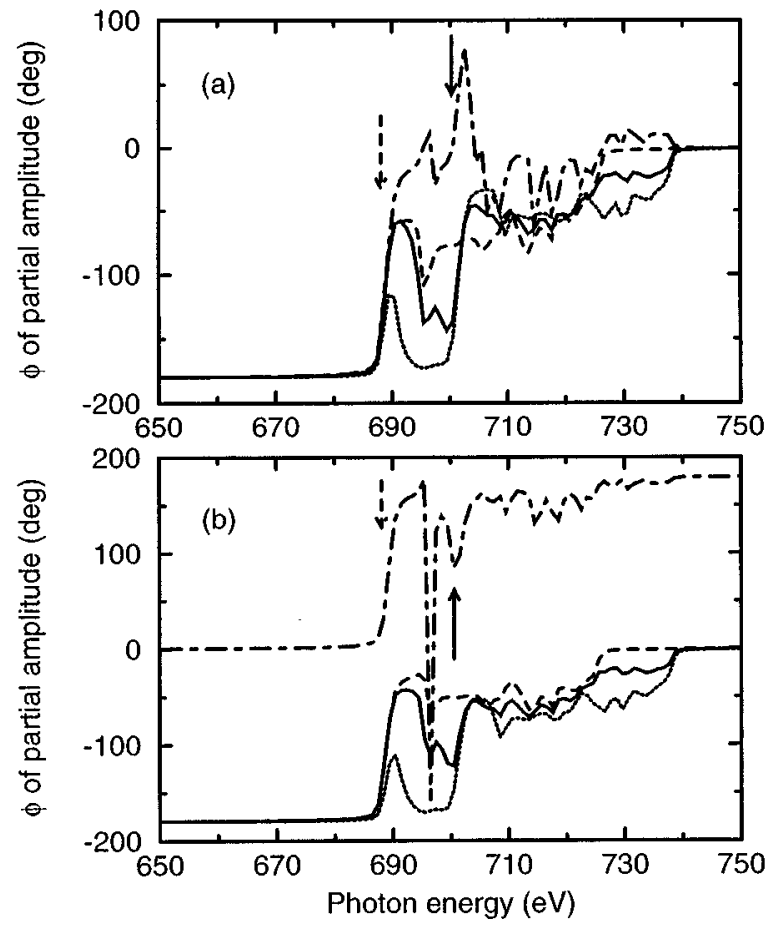

FIG. 4. Phase behavior of the partial wave contributions to the scattering amplitude at the iron $L_{\mathrm{II}}$ and $L_{\mathrm{III}}$ absorption edges. For further details, see the caption to Fig. 3.

Evidently, all features of interest in the scattering cross section of Fig. 5 (denoted as symbols $A, B$, and $C$ ) can be qualitatively explained in terms of the squared $p$-projected density of states above the Fermi level. Computationally this is due to the following reasons. The matrix elements of Eq. (20c) are in general smooth functions of the band energy $\epsilon$. Therefore, most structure in the scattering cross section comes from the behavior of the $\operatorname{Im} \tau_{\Lambda \Lambda^{\prime}}^{00}(\epsilon)$ elements of Eq. (20b). The selection rules for our X-ray-beam geometry are in $E 1$ approximation simply: $l_{0}-l= \pm 1, \mu=\mu_{0}+1$ for LCP radiation, and $\mu=\mu_{0}-1$ for RCP radiation. Therefore, only $p$ states related $\operatorname{Im} \tau^{00}(\epsilon)$ elements are responsible for the $\epsilon$ dependence of the numerator part of Eq. (20b). Then, by virtue of the multiple-scattering expression for the Green's function by Faulkner and Stocks, ${ }^{27}$ it can been seen that the numerator part of Eq. (20b) is approximately proportional to the $p$-projected density of states. As it can be seen from Fig. 5 , the calculated scattering cross section closely follows the features of the square of the normalized $p$ density of states.

On the other hand, for the $L_{\mathrm{II}, \mathrm{III}}$-edge resonant scattering we find that the scattering cross section does not reproduce the rapidly varying features of the $d$ density of states above the Fermi level. This is because in this case the $\Gamma$ parameter $(1.0 \mathrm{eV})$ is large enough to wash out the sharp features when the integration over $\epsilon$ is carried out in Eq. (20b). And as a result only one pronounced peak will appear in the scattering spectra nearby each absorption edge.

These important observations have the general implication that in favorable conditions the measured resonant $\mathrm{x}$-ray-scattering spectra contain much spectroscopic information about the partial density of states in the unoccupied part

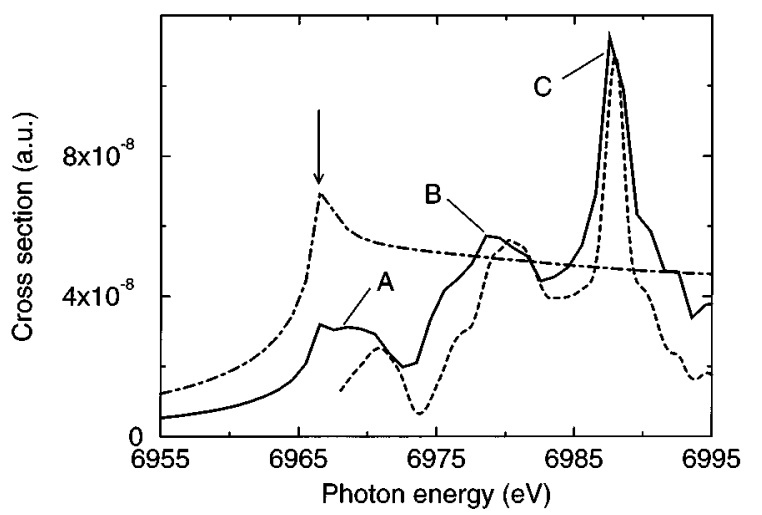

FIG. 5. Calculated electric dipole (E1) contribution to the anomalous scattering cross section at the iron $K$ absorption edge (solid line). The incoming and scattered photons propagate along the magnetization axis. The squared $p$-projected density of states (dashed line) has been normalized according to the maximum value of peak $C$. In order to notice the dramatic difference between the crystalline and atomic case the single-site scattering-derived cross section has been presented (dash-dotted line). The arrow locates the position of the $K$ absorption edge.

of the band structure, illustrating that band structure effects are important for understanding resonant magnetic features of $\mathrm{x}$ rays.

There is yet another remarkable finding which we demonstrate within our calculations. Namely, in addition to the full crystalline scattering cross section which we have discussed above, we have repeated the same calculation at the $K$ absorption edge of iron for an isolated muffin-tin potential by using a single-site scattering matrix $t^{0}(\epsilon)$ in place of the path operator $\tau^{00}(\epsilon)$ in Eq. (20b). Although this only mimics the real atomic resonant scattering, we can immediately see from Fig. 5 that the multiple-scattering effects drastically alter the spectral behavior of the scattering cross section. The flat behavior of $p$-derived $\operatorname{Im} t^{0}(\boldsymbol{\epsilon})$ elements above the Fermi level is responsible for the single peak in the "atomic" scattering cross section, while the overlapping $p$ wave functions via the path operator $\tau^{00}$ are responsible for the cross section in iron crystal. By contrast, at the $L_{\mathrm{II}^{-}}$and $L_{\mathrm{III}}{ }^{-}$edge resonant scattering there is only a small difference between these two cases. This is because the electric dipole transitions $(E 1)$ involve now intermediate $d$ states, which are more localized than the $p$ states, and consequently multiple-scattering effects are small.

To complete the discussion about the $K$-edge resonant scattering of iron one may ask whether the higher multipole contributions are important. To answer this question we show the $(M 1+E 2)$ contribution to the anomalous scattering cross section together with the single-site (atomic) scattering spectra and the $d$-projected density of states in Fig. 6 . As is clear from Fig. 6, the overall $(M 1+E 2)$ contribution to the scattering cross section is about 100 times smaller to the electric dipole $(E 1)$ contribution of Fig. 5. However, there are some qualitatively interesting features in Fig. 6. In accordance with the the $(M 1+E 2)$ selection rules given in the end of Sec. II D 2 the $d$ states above the Fermi level build up the large resonant peak at the $K$ edge. Interestingly, the $(M 1+E 2)$ contribution to the scattering cross section of 


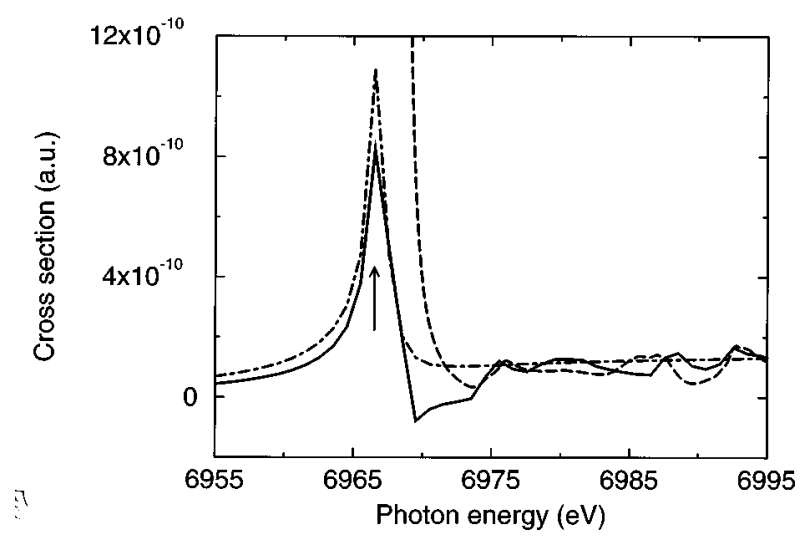

FIG. 6. Calculated $(M 1+E 2)$ contribution to the anomalous scattering cross section at the iron $K$ absorption edge (solid line). A properly normalized, Lorentzian lifetime-broadened $(\Gamma=0.8 \mathrm{eV})$ and squared $d$-projected density of states (dashed line) and a singlesite scattering-derived cross section (dash-dotted line) have been also presented. The arrow locates the position of the $K$ absorption edge.

Fig. 6 possesses similar oscillations as the Lorentzian lifetime-broadened $(\Gamma=0.8 \mathrm{eV})$ and squared $d$-projected density of states. This further substantiates the useful link between the resonant scattering spectra and the partial density of states in the empty part of the band structure. Also we notice from Fig. 6 that in the single-site scattering case the $(M 1+E 2)$ contribution to the cross section is very similar to that in the crystalline case near the $K$ absorption edge. As can be inferred from the $L_{\mathrm{II}, \mathrm{III}}$-edge resonant scattering case above this is solely due to the localized nature of the $d$ states above the Fermi level.

\section{Dichroism at $L_{\mathrm{II}}$ and $L_{\mathrm{III}}$ edges}

Since the dichroism in the resonant magnetic scattering at the $K$ edge of iron (exclusively $E 1$ derived) is about 50 times smaller than the dichroism at the $L_{\text {II,III }}$ edges, we limit our study here solely to the $L_{\mathrm{II}, \mathrm{III}}$ edges. The magnetic x-rayscattering dichroism at $L_{\mathrm{II}, \mathrm{III}}$ edges is in general a delicate combined effect of spin-orbit interaction and spin polarization in the core states and the intermediate states. However, by looking again at the numerator of Eq. (20b) we can easily envisage the microscopic origin of the various features of the dichroism spectra of Fig. 7.

The magnitude of the first important matrix element of the transition $\left(p_{1 / 2},-1 / 2\right) \rightarrow\left(d_{3 / 2},+1 / 2\right)$ contributing to the $L_{\mathrm{II}}$-derived peak $B_{+}$of Fig. 2 for $\mathrm{LCP}$ radiation is nearly equal to the magnitude of the transition $\left(p_{1 / 2},+1 / 2\right) \rightarrow\left(d_{3 / 2},-1 / 2\right)$ contributing to the peak $B_{-}$ for RCP radiation. Similarly, the magnitude of the second important matrix element of the transition $\left(p_{1 / 2},+1 / 2\right) \rightarrow\left(d_{3 / 2},+3 / 2\right)$ contributing to the $L_{\mathrm{II}}$-derived peak $B_{+}$of Fig. 2 for LCP radiation is nearly equal to the magnitude of the transition $\left(p_{1 / 2},-1 / 2\right) \rightarrow\left(d_{3 / 2},-3 / 2\right)$ contributing to the peak $B_{-}$for RCP radiation.

Therefore we can conclude that the feature $B$ of Fig. 7 is approximately proportional to the differences $\operatorname{Im} \tau_{2+1 / 2 ; 2+1 / 2}^{00}(\boldsymbol{\epsilon})-\operatorname{Im} \tau_{2-1 / 2 ; 2-1 / 2}^{00}(\boldsymbol{\epsilon})$ and $\operatorname{Im} \tau_{2+3 / 2 ; 2+3 / 2}^{00}(\boldsymbol{\epsilon})$ $-\operatorname{Im} \tau_{2-3 / 2 ; 2-3 / 2}^{00}(\epsilon)$ in the standard $\kappa \mu$ representation ${ }^{20}$

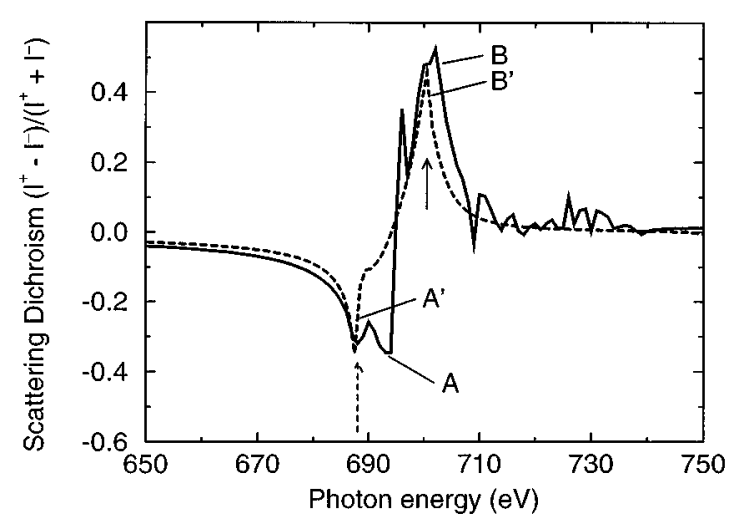

FIG. 7. The calculated asymmetry ratio (solid line) in the electric dipole approximation together with the asymmetry ratio (dashed line) computed in the atomic limit. The symbols $A, A^{\prime}, B$, and $B^{\prime}$ are discussed in the text.

nearby the Fermi level, where there is a large, unoccupied $d$ density-of-states peak. Because these differences vanish in a non-spin-polarized relativistic case, the dichroism feature $B$ can be interpreted purely in terms of the exchange splitting in the $d_{3 / 2}$-like intermediate states near the Fermi level. As can be deduced from Fig. 3 , the $d_{5 / 2}$-like states have only a minor contribution to the feature $B$ of Fig. 7 .

Similarly when analyzing the $L_{\mathrm{III}}$-edge-derived dichroism feature $A$ of Fig. 7 we find that the exchange splitting of $d_{5 / 2}$-like states is mostly responsible for that feature. However, it is interesting to notice that the $\left(d_{3 / 2}, d_{5 / 2}\right)$-like spinflip elements of the $\tau^{00}$ matrix, which tend to diminish the scattering amplitude for the RCP radiation (see the discussion in Sec. III A), reduce to a large extent the magnitude of the feature $A$. On the other hand, as can be deduced by looking at the magnitude (Fig. 3) and phase (Fig. 4) behavior of the partial scattering amplitudes, the diagonal $d_{5 / 2}$ and $\left(d_{3 / 2}, d_{5 / 2}\right)$ spin-flip elements of $\tau^{00}$ make hardly any contribution to peaks $B_{+}$and $B_{-}$. Therefore, the dichroism feature $B$ of Fig. 7 is solely due to the diagonal $d_{3 / 2}$ elements of $\tau^{00}$.

It can also be observed from Fig. 7 that the crystalline (multiple-scattering) effects do not change the qualitative picture of the dichroism spectra from the atomic (single-site scattering) case. This is in agreement with the computed scattering cross sections at the $L_{\text {II,III }}$ edges for the crystalline and atomic cases (see the discussion in the end of Sec. III B).

As a very important technical aspect of our calculation we note that due to the overlapping feature of the $L_{\mathrm{II}^{-}}$and $L_{\mathrm{III}}$-derived scattering peaks of Fig. 2 it is vital to compute the $L_{\mathrm{II}}$ and $L_{\mathrm{III}}$ contributions to the scattering amplitude simultaneously and add coherently. If they are calculated separately, the dichroism (the sum of the dashed and dasheddotted curves of Fig. 8) will not be evaluated correctly as Fig. 8 shows. Notably, the correct positions of the $L_{\mathrm{III}^{-}}$and $L_{\mathrm{II}}$-related features $A^{\prime}$ and $B^{\prime}$ of Fig. 8 notwithstanding, their intensities do not reproduce the peaks $A$ and $B$ correctly.

Our calculated spectral features of dichroism agree qualitatively with those measured by Kao et al., ${ }^{7}$ the main difference being that the largest asymmetry ratio, which comes from the $L_{\mathrm{II}}$-edge resonance, is $13 \%$ in their measurement 


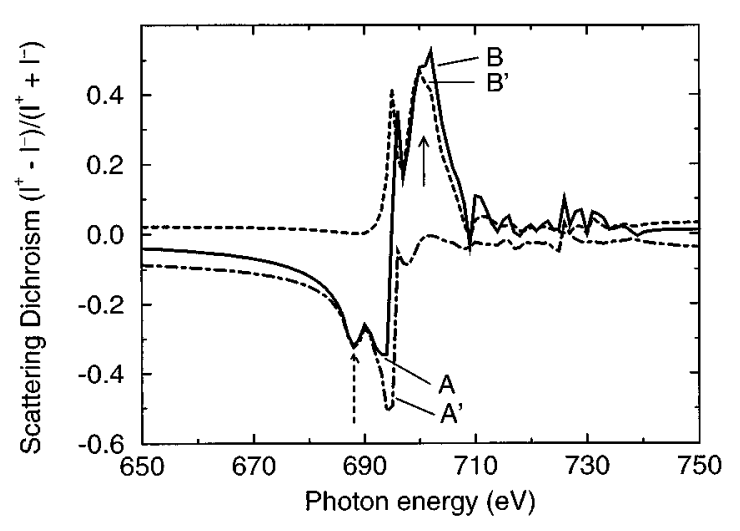

FIG. 8. The calculated asymmetry ratio (solid line) in the electric dipole approximation in the case where both $L_{\mathrm{II}}$ and $L_{\mathrm{III}}$ core states have been used for the scattering amplitude. Also shown are the asymmetry ratios produced using only $L_{\mathrm{II}}$ (dashed line) or $L_{\mathrm{III}}$ (dash-dotted line) core states in the scattering amplitude formula. The symbols $A, A^{\prime}, B$, and $B^{\prime}$ are discussed in the text.

while the corresponding value according to our calculation is about $50 \%$. This discrepancy can be mainly attributed to the following facts. We have used completely circularly polarized $\mathrm{x}$ rays in our calculations for the bulk iron whereas Kao et al. have used $p$-polarized light scattered within a different geometry (specular reflection) from a single-crystal iron film. Also, we have ignored the absorption and experimental broadening effects in our calculation. Including them would significantly decrease the calculated asymmetry ratio.

\section{CONCLUSIONS}

We have presented a detail derivation of a first-principles formalism for magnetic scattering of circularly polarized $\mathrm{x}$ rays from magnetic solids which enables us to calculate not only the magnetic structural information, but also to interpret the microscopic nature of various spectral features in the scattering cross section and the dichroism curves. We have demonstrated this by studying resonant magnetic x-ray scattering at $L_{\text {IIIIII }}$ and $K$ absorption edges of iron. Especially we have noticed that multiple-scattering effects have an important contribution to the $\mathrm{x}$-ray resonant scattering cross section at the $K$ edge. On the other hand, at the $L_{\text {II.III }}$ edges there occur only minor changes due to the more localized nature of the $d$ electrons. Furthermore, if the lifetime effects are small enough, then the $\mathrm{x}$-ray resonant scattering measurement allows us to study the partial density-of-states features of the empty part of the band structure above the Fermi level in addition to information about the magnetic structure encoded in the position of the magnetic Bragg peaks. We have demonstrated this by computing the x-ray resonant scattering cross section at the $K$ absorption edge, and compared this with the squared value of the $p$ partial density of states. The agreement between these is amazingly good.

Being fully relativistic, our formalism treats spin-orbit coupling and spin-polarization effects on an equal footing and hence gives an account of the $\mathrm{x}$-ray magnetic circular dichroism (XMCD) in magnetic scattering. A fully relativistic calculation is also important in the case of systems containing heavy elements where the spin-orbit interaction cannot be treated as a simple perturbation, as in the cases of lanthanide or actinide materials where the higher-order multipole transitions become also important.

Finally we want to emphasize that in addition to being able to include the crystalline environmental contribution to the scattering amplitude accurately, the above multiplescattering theory for the electron Green's function, Eq. (12), allows us to make a site decomposition of the general scattering formula, Eq. (17). Therefore applying the above magnetic x-ray resonant scattering formalism it will be possible to focus on studying site-resolved magnetic moment correlations in such technologically important alloys as $\mathrm{Ni}_{c} \mathrm{Fe}_{1-c}$.

\section{ACKNOWLEDGMENTS}

One of us (E.A.) is indebted to the Academy of Finland and the Royal Society, under whose auspices part of this work has been carried out at the University of Bristol.
${ }^{1}$ F. E. Low, Phys. Rev. 96, 1428 (1954); M. Gell-Mann and M. L. Goldberger, ibid. 96, 1433 (1954).

${ }^{2}$ P. M. Platzman and N. Tzoar, Phys. Rev. B 2, 3556 (1970).

${ }^{3}$ M. Blume, J. Appl. Phys. 57, 3615 (1985).

${ }^{4}$ K. Namikawa, M. Ando, T. Nakajima, and H. Kawata, J. Phys. Soc. Jpn. 54, 4099 (1985).

${ }^{5}$ F. de Bergevin and M. Brunel, Acta Crystallogr. Sect. A 37, 314 (1981)

${ }^{6}$ D. Gibbs, D. R. Harshman, E. D. Isaacs, D. B. McWhan, D. Mills, and C. Vettier, Phys. Rev. Lett. 61, 1241 (1988).

${ }^{7}$ C. Kao, J. B. Hastings, E. D. Johnson, D. P. Siddons, G. C. Smith, and G. A. Prinz, Phys. Rev. Lett. 65, 373 (1990).

${ }^{8}$ C.-C. Kao, C. T. Chen, E. D. Johnson, J. B. Hastings, H. J. Lin, G. H. Ho, G. Meigs, J.-M. Brot, S. L. Hulbert, Y. U. Idzerda, and C. Vettier, Phys. Rev. B 50, 9599 (1994).

${ }^{9}$ M. Blume and D. Gibbs, Phys. Rev. B 37, 1779 (1988).

${ }^{10}$ J. P. Hannon, G. T. Trammel, M. Blume, and D. Gibbs, Phys. Rev. Lett. 61, 1245 (1988).
${ }^{11}$ A. Fasolino, P. Carra, and M. Altarelli, Phys. Rev. B 47, 3877 (1993).

${ }^{12}$ P. Rennert, Phys. Rev. B 48, 13559 (1993).

${ }^{13}$ J. C. Lang, G. Srajer, C. Detlefs, A. I. Goldman, H. König, X. Wang, B. N. Harmon, and R. W. McCallum, Phys. Rev. Lett. 74, 4935 (1995).

${ }^{14}$ C. Giorgetti, E. Dartyge, C. Brouder, F. Baudelet, C. Meyer, S. Pizzini, A. Fontaine, and R-M. Galéra, Phys. Rev. Lett. 75, 3186 (1995).

${ }^{15}$ J. P. Hill, A. Vigliante, D. Gibbs, J. L. Peng, and R. L. Greene, Phys. Rev. B 52, 6575 (1995).

${ }^{16}$ P. Carra, M. Altarelli, and F. de Bergevin, Phys. Rev. B 40, 7324 (1989).

${ }^{17}$ P. Strange, H. Ebert, J. B. Staunton, and B. L. Gyorffy, J. Phys. Condens. Matter 1, 2959 (1989).

${ }^{18}$ P. Strange, J. Staunton, and B. L. Gyorffy, J. Phys. C 17, 3355 (1984).

${ }^{19}$ E. Arola, in Acta Polytechnica Scandinavica, Applied Physics 
Series No. 174, edited by M. Luukkala (The Finnish Academy of Technology, Helsinki, 1991).

${ }^{20}$ J. J. Sakurai, Advanced Quantum Mechanics (Addison-Wesley, Reading, MA, 1967).

${ }^{21}$ P. J. Durham, in Electronic Structure of Complex Systems, edited by P. Phariseau and W. M. Temmerman, Vol. 113 of NATO Advanced Study Institute, Series B: Physics (Plenum Press, New York, 1984).

${ }^{22}$ L. V. Keldysh, Sov. Phys. JETP 20, 1018 (1965).

${ }^{23}$ It is noticeable that $v_{\Lambda}(\vec{r})$ represents a real positron $e^{+}$, which can closely be related to one of the negative-energy electron states by symmetry of the Dirac equation.

${ }^{24}$ In the nonrelativistic case both the first- and second-order terms of Eq. (1) contribute to the scattering amplitude (Ref. 3).

${ }^{25}$ Our definition of the scattering amplitude is identical to the commonly used concept of "matrix element" [see, for example, Rennert (Ref. 12)], and therefore is slightly different (see discussion at the end of Sec. II E) from the standard one $[d \sigma / d \Omega$ $\equiv|f(\omega)|^{2}$, where $d \sigma / d \Omega$ is the differential scattering cross section and $f(\omega)$ is the scattering amplitude]. By doing this, we avoid confusion with another "matrix element" concept in our theory (see Sec. II D). Our scattering amplitude results, however, are presented in terms of the standard scattering amplitude (see Figs. 3 and 4).

${ }^{26}$ We basically need the Green's function $\mathcal{G}^{+}\left(\vec{r}, \vec{r}^{\prime}, \epsilon\right)$ only for $\vec{r}$ and $\vec{r}^{\prime}$ both lying within the same site, because the core states $u_{\Lambda}(\vec{r})$ in Eq. (11) are usually highly localized inside a given muffin-tin sphere.

${ }^{27}$ J. S. Faulkner and G. M. Stocks, Phys. Rev. B 21, 3222 (1980).

${ }^{28}$ A. H. MacDonald and S. H. Vosko, J. Phys. C 12, 2977 (1979).
${ }^{29}$ Therefore, the solution $Z_{\Lambda}\left(\vec{r}_{n}, \epsilon\right)$ [and similarly $J_{\Lambda}\left(\vec{r}_{n}, \epsilon\right)$ ] does not have a well-defined spin angular character, and only $\mu$ remains as a good quantum number (Ref. 30).

${ }^{30}$ H. Ebert, B. Drittler, P. Strange, R. Zeller, and B. L. Gyorffy, in The Effects of Relativity on Atoms, Molecules, and the Solid State, edited by S. Wilson et al. (Plenum Press, New York, 1991).

${ }^{31}$ V. L. Moruzzi, J. F. Janak, and A. R. Williams, Calculated Electronic Properties of Metals (Pergamon, Oxford, 1978).

${ }^{32}$ D. D. Johnson, F. J. Pinski, and G. M. Stocks, J. Appl. Phys. 57, 3018 (1985).

${ }^{33}$ M. E. Rose, Relativistic Electron Theory (Wiley, New York, 1961).

${ }^{34}$ G. Schütz, W. Wagner, W. Wilhelm, P. Kienle, R. Zeller, R. Frahm, and G. Materlik, Phys. Rev. Lett. 58, 737 (1987).

${ }^{35}$ O. Keski-Rahkonen and M. O. Krause, At. Data Nucl. Data Tables 14, 139 (1974).

${ }^{36}$ If an atomic $L_{\mathrm{II}, \mathrm{III}}$-subshell core-hole lifetime value of $\Gamma=0.24$ $\mathrm{eV}$ is used, then the dichroism curve (with or without an extra Gaussian instrumental broadening convolution on the cross section) contains quite profound oscillations, which are not present in the measured dichroism curves provided by Kao et al. (Ref. 7). Furthermore, using $\Gamma=1 \mathrm{eV}$ reproduces well all the features observed in the measured dichroism curve by Kao et al. (Ref. 7 ). We have also noticed that using $\Gamma$ values larger than about 1 $\mathrm{eV}$ will not change the qualitative nature of the dichroism curve. We want to stress that $\Gamma$ is just a parameter reflecting the manybody effects involved in the $\mathrm{x}$-ray-scattering process, and is not linked in any systematic manner to the $a b$ initio part of the theory, i.e., to the LDA electronic structure. 